\title{
Multiharmonic vector rf voltage control for wideband cavities driven by vacuum tube amplifiers in a rapid cycling synchrotron
}

\author{
Fumihiko Tamura $\odot^{*}$ Yasuyuki Sugiyama, Masahito Yoshii, Masanobu Yamamoto, \\ Chihiro Ohmori, Masahiro Nomura, Taihei Shimada, Katsushi Hasegawa, \\ Keigo Hara, and Masashi Furusawa \\ J-PARC Center, JAEA and KEK, 2-4 Shirakata, Tokai, Naka, Ibaraki, Japan 319-1195
}

(Received 17 April 2019; published 26 September 2019)

\begin{abstract}
Beam loading compensation in the rf cavities is a key for acceleration of high-intensity beams in the $3 \mathrm{GeV}$ rapid cycling synchrotron (RCS) of the Japan Proton Accelerator Research Complex (J-PARC). Since we employ wideband magnetic alloy rf cavities for the J-PARC RCS and the wake voltage contains several harmonics, multiharmonic beam loading compensation is required. The multiharmonic if feedforward for the six most important harmonics is implemented in the existing low-level rf (LLRF) control system, which has been working fairly well for acceleration of high-intensity beams of up to $1 \mathrm{MW}$. However, we found degradation of the performance for compensation of the feedforward with very-highintensity beams. The gain variation with output current of the tube amplifier affects the performance of the feedforward, since it has an open loop configuration. Also, there is a voltage waveform distortion caused by the distortion of the output current, which essentially cannot be compensated by the feedforward. Therefore, we consider employing the vector rf voltage feedback control for beam loading compensation, where the feedback is expected to work with the gain variation within the stability margin. A multiharmonic vector rf voltage control has been developed as the core part of the next-generation LLRF control system for RCS. Prior to the completion of the new system, the multiharmonic vector rf voltage control was tested with one of the 12 cavities. The details of the system configuration, commissioning methodology, and beam test results using very-high-intensity beams are described. Beam loading by the $1 \mathrm{MW}$ equivalent beam in the cavity is successfully compensated.
\end{abstract}

DOI: 10.1103/PhysRevAccelBeams.22.092001

\section{INTRODUCTION}

The $3 \mathrm{GeV}$ rapid cycling synchrotron (RCS) of the Japan Proton Accelerator Research Complex (J-PARC) $[1,2]$ is a high-intensity proton synchrotron, which simultaneously delivers proton beams to the Material and Life Science Experimental Facility (MLF) and the main ring synchrotron (MR).

The main parameters of the RCS and its rf system are listed in Table I. The design beam intensity is $8.3 \times 10^{13}$ protons per pulse (ppp), corresponding to the output beam power of $1 \mathrm{MW}$ at the repetition rate of $25 \mathrm{~Hz}$. The output beam power of the RCS has been steadily increased with hardware upgrades and beam tuning since the RCS beam operation was started in October 2007. At present, the routine output beam power for the user program of the MLF

\footnotetext{
*fumihiko.tamura@j-parc.jp

Published by the American Physical Society under the terms of the Creative Commons Attribution 4.0 International license. Further distribution of this work must maintain attribution to the author(s) and the published article's title, journal citation, and DOI.
}

is $500 \mathrm{~kW}$. High-intensity beam tests up to the design beam intensity of $8.3 \times 10^{13}$ ppp were performed, and we established $1 \mathrm{MW}$ beam acceleration with a very low fractional beam loss below $0.2 \%$ [3] in the single-shot mode, where a beam macropulse is injected from the linac to the RCS on demand. Also, we successfully demonstrated $1 \mathrm{MW}$ continuous operation at the repetition rate of $25 \mathrm{~Hz}$ for one hour in July 2018.

For high-intensity proton synchrotrons and storage rings, beam loading [4-6] due to the wake voltage in the cavity

TABLE I. Parameters of the J-PARC RCS and its rf system.

\begin{tabular}{lc}
\hline \hline Circumference & $348.333 \mathrm{~m}$ \\
Energy & $0.400-3 \mathrm{GeV}$ \\
Beam intensity & $8.3 \times 10^{13} \mathrm{ppp}$ \\
Output beam power & $1 \mathrm{MW}$ \\
Accelerating frequency & $1.227-1.671 \mathrm{MHz}$ \\
Harmonic number & 2 \\
Maximum rf voltage & $440 \mathrm{kV}$ \\
Repetition rate & $25 \mathrm{~Hz}$ \\
No. of cavities & 12 \\
$Q$ value of rf cavity & 2 \\
\hline \hline
\end{tabular}


induced by the beam must be considered carefully. Beam loading compensation is a key as well to mitigation of space charge effects.

The beam loading at the accelerating harmonic must be compensated to keep the stability of the motion of the rigid bunch [5]. The relative loading factor $Y=I_{b} / I_{0}$ [5], where $I_{b}$ is the beam current and $I_{0}$ is the resistive current into the cavity, for the $1 \mathrm{MW}$ beam acceleration with the normal voltage pattern varies from $Y=0.8$ to $Y=6.9$. It reaches to an especially high value at 6.9 just before extraction, where the programed voltage is low. Thus, beam loading compensation at the accelerating harmonic is indispensable.

Also, cavity impedance at the revolution harmonics can be a source of longitudinal coupled bunch instabilities [7-10], which may introduce unwanted longitudinal emittance growth. According to our analysis [11,12], longitudinal coupled bunch instabilities are not expected to appear in the J-PARC RCS.

Bucket distortion [13-15] in wideband magnetic alloy (MA) cavities $[16,17]$ due to the higher harmonics of the wake voltages is a relatively new topic, since the application of MA cavities to high-intensity synchrotrons was pioneered by the J-PARC constructed in this century. Bucket distortion affects the longitudinal distribution and is an issue on the bunch shape and emittance control. The MA cavity in the J-PARC RCS has a wideband frequency response with a low $Q$ value of 2, which covers the accelerating frequency sweep from 1.228 to $1.671 \mathrm{MHz}$ with the velocity change of the proton beam. It enables also dual harmonic operation, in which a single cavity is driven by the superposition of the fundamental accelerating harmonic $(h=2)$ and the second harmonic $(h=4)$ for bunch shaping. The wake voltage in the MA cavity contains several harmonics, where the amplitudes of up to the third harmonic $(h=6)$ are comparable to the voltage program of the fundamental accelerating harmonic. Multiharmonic beam loading compensation against bucket distortion is necessary.

The rf system does not have fast rf feedback around the final stage amplifier, which is considered to be one of the most powerful beam loading compensation schemes and widely employed in many proton synchrotrons and storage rings in the world [18-21]. Fast feedback with the low $Q$ value of 2 requires a very low latency of the feedback path, less than $30 \mathrm{~ns}$ according to the theory [6], which is not easy to realize. We decided not to have fast rf feedback in the RCS rf system at the time of construction, while we should note here that a successful application of fast $\mathrm{rf}$ feedback for a wideband MA cavity using a solid-state amplifier at CERN was reported recently $[22,23]$. Therefore, beam loading compensation is implemented in the low-level rf (LLRF) control system of the RCS.

As described in our previous articles [24,25], we employ the rf feedforward method [26,27] for multiharmonic beam loading compensation in the wideband

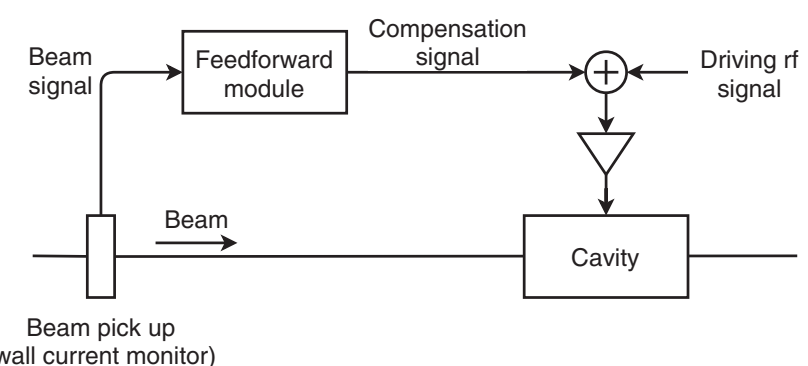

(wall current monitor)

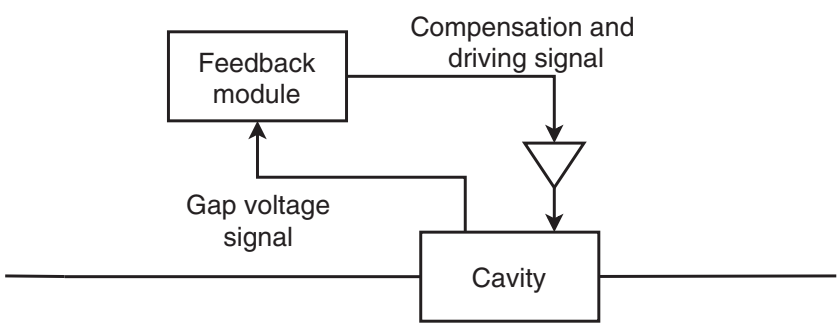

FIG. 1. Conceptual diagrams of (top) the rf feedforward method and (bottom) the voltage feedback.

MA cavity in the RCS. A conceptual diagram of the rf feedforward method is illustrated in the top of Fig. 1. The compensation signal is generated by using the beam signal so that the wake voltage is canceled.

The multiharmonic feedforward system compensates the beam loading of the fundamental accelerating harmonic $(h=2)$ and the higher harmonics up to the third harmonics $(h=6)$. The odd harmonics $(h=1,3,5)$ are compensated for high-intensity single-bunch acceleration.

The feedforward system works fairly well for highintensity operation with up to $1 \mathrm{MW}$ equivalent beams. The acceleration is stable and the unwanted beam oscillation is not observed, which implies that the compensation at the fundamental acceleration keeps the stability condition within the limit, and no beam losses at the dispersion peaks due to the longitudinal motion are observed. However, we found that the compensation at more than $600 \mathrm{~kW}$ is not as good as in our previous articles, in which the beam tests were performed with beams at less than $400 \mathrm{~kW}$. The phase of the rf cavity voltage and then the beam phase with respect to the reference phase signal vary with various beam intensities at more than $600 \mathrm{~kW}$, which implies that the compensation at the fundamental accelerating harmonic is not very good. (See Sec. III B 4 in Ref. [24]). We have to align the rf phase of the MR so that the bunch is injected to the center of the MR rf bucket, when the injected beam intensity is changed for beam commissioning. Compensation of the higher harmonics is not as good as the previous article [24]. For example, suppression of better than $30 \mathrm{~dB}$ of the second harmonic with a $300 \mathrm{~kW}$ equivalent beam is reported in Ref. [24], but suppression of only $1 / 4$ to $1 / 3$, which varies during the cycle, is observed when accelerating the $1 \mathrm{MW}$ beam. The voltage waveform is clearly distorted especially near extraction, where the programed voltage is low. The voltage 
waveform distortion introduces a discrepancy of the bunch shape between the real beams and the transverse space charge tracking simulations, where the beam loading effects are hardly implemented. The discrepancy affects the transverse beam loss prediction by the simulation. Although stable acceleration of the beams at the design intensity is achieved with the existing feedforward system, better beam loading compensation is demanded.

The feedforward compensation signals are generated from the beam signal. In our commissioning methodology $[24,25]$, we assume linearity of the system. For the veryhigh-intensity beams, the nonlinearity of the vacuum tube amplifier degrades the performance of the compensation. Furthermore, the voltage waveform distortion due to the distortion of the tube amplifier output current becomes intolerable under heavy beam loading, which requires a high tube output current for compensation.

In summary, our conclusion of the previous articles $[24,25]$ that the multiharmonic feedforward would work at the design beam power of $1 \mathrm{MW}$ as lower beam power is based on an underestimation of the nonlinearity of the amplifier chain. At a very high beam power, we found that the effects of the nonlinearity are significant and severe.

Therefore, we consider employing the vector rf voltage feedback control for beam loading compensation. As illustrated in the bottom of Fig. 1, the feedback picks up the gap voltage signal to generate the compensation and driving signal. Because of its closed loop configuration, it is expected that the feedback can compensate the beam loading with the gain variation of the tube amplifier as long as the variation is within the stability margin. Also, the feedback can reduce the waveform distortion due to the tube current distortion. These effects are not significant for the acceleration of beams at less than $400 \mathrm{~kW}$.

A multiharmonic vector $r f$ voltage control was developed as the core part of the next-generation LLRF control system for the RCS. The existing LLRF control system, which has been in operation for more than 10 years, is to be replaced with a new system in the near future. Prior to the completion of the new system, the multiharmonic vector rf voltage control was extensively tested.

In this article, we describe the development of the multiharmonic vector if control. First, we investigate the nonlinearity of the beam loading compensation by using a circuit simulator. Also, the in situ measurement of the solid-state amplifier when accelerating a high-intensity beam is presented. We describe the configuration of the system. The commissioning and characterization of the system are described. We performed beam tests with very high power beams up to $1 \mathrm{MW}$ equivalent. The various beam test results are reported.

\section{NONLINEARITY OF BEAM-LOADING COMPENSATION}

Circuit simulations using LTspice [28] are performed to investigate the nonlinearity of beam loading compensation.
The simplified circuit model used for the simulations including the cavity, amplifier, and beam current is shown in Fig. 2. The MA cavity is modeled as a parallel $L C R$ circuit with a single accelerating gap, while the real cavity has three accelerating gaps [29], ignoring the inductance of the bus bars between three gaps. A parallel inductor [30] and a tuning capacitor are connected to the accelerating gap for adjusting the resonant frequency and $Q$ value of the cavity. The circuit constants are set so that the cavity resonance is reproduced. Contrary to the ferrite cavity with a tuning bias loop to follow the change of the rf frequency, the MA cavity is regarded as a broadband passive load. The circuit constants are fixed for different rf frequencies.

The amplifier has two tetrode vacuum tubes, Thales TH558K, which are connected at the upstream and downstream of the accelerating gap via dc blocking capacitors. This is a class AB push-pull amplifier, where the two tubes supply current to the cavity alternately. The tetrode model of TH558K is provided by CERN. The tetrodes are modeled using behavioral model current generators. The response is derived mathematically, fitting the constant current curves from the data sheet at a fixed screen grid voltage [31]. The control grid of the tube is driven by a single harmonic rf voltage source in the simulation, while it is driven by a solid-state amplifier and a splitter in the real system. The screen grid voltage is set to $1.75 \mathrm{kV}$, and the idling current of the each tube is adjusted to $5 \mathrm{~A}$ by setting the dc voltage of the control grid.

A single harmonic rf current source is connected to the accelerating gap to simulate the beam current, while the real beam contains higher harmonics. A factor of 3 is applied, since the three gaps are simulated by using a single gap. The current supplied by the current source is

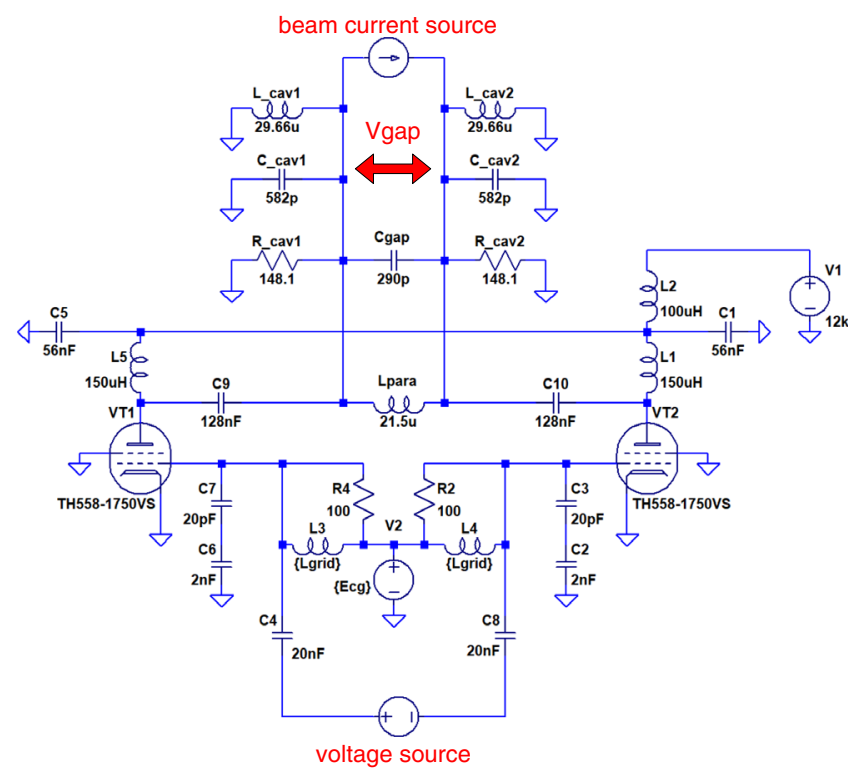

FIG. 2. Circuit model including the cavity, amplifier, and beam. 

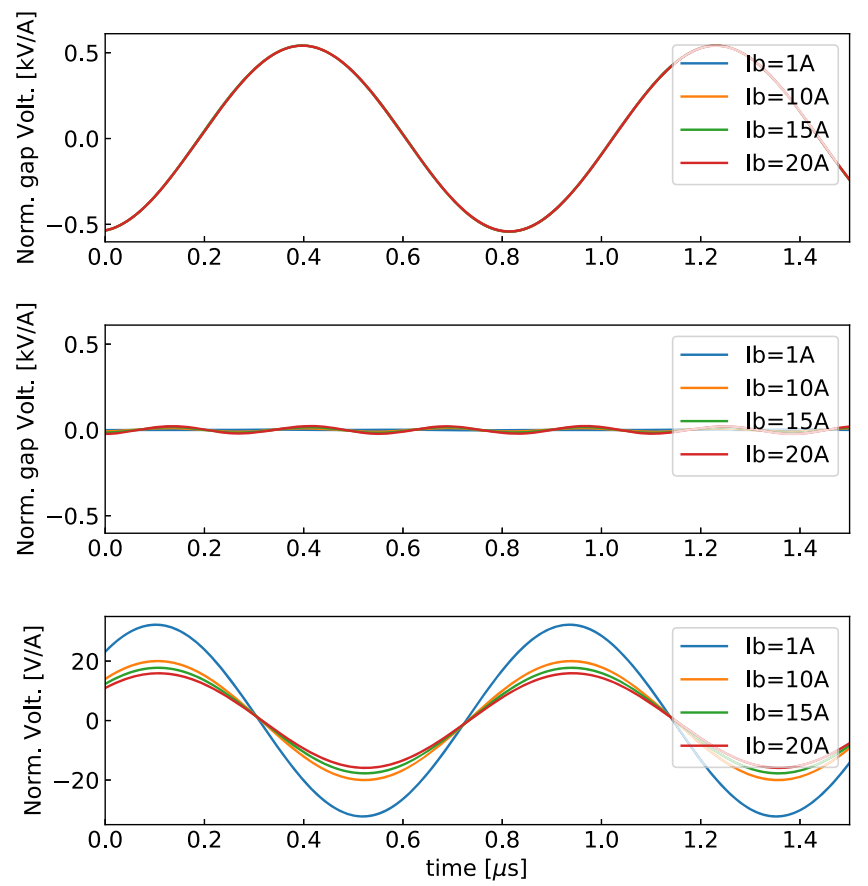

FIG. 3. (Top) Gap voltage waveforms normalized by the beam current without compensation, (middle) gap voltage waveforms normalized by the beam current with compensation by adjusting the amplitude and phase of the rf voltage source, and (bottom) normalized output waveform of the voltage source for compensation.

$$
\text { ( supplied current })=3 \times I_{b} \sin \left(2 \pi f_{\mathrm{rf}} t+\phi_{b}\right),
$$

where $I_{b}$ and $\phi_{b}$ are the amplitude and phase, respectively, of the beam current, $f_{\mathrm{rf}}$ is the rf frequency, and $t$ is the time. $f_{\text {rf }}=1.2 \mathrm{MHz}$, which is close to the injection frequency, is chosen for the simulation.

The impedance seen by the beam consists of not only the cavity but also the amplifier including the tubes. The impedance of the tube varies with its operating condition.

The wake voltage at the accelerating gap is simulated by switching off the voltage source, where the tubes are idling. The normalized gap voltages $V_{\text {gap }} / I_{b}$ with various beam currents, where $V_{\text {gap }}$ is defined as the differential voltage between the upstream and downstream of the accelerating gap, are plotted in the top of Fig. 3. The normalized gap voltages with the beam current from 1 to $20 \mathrm{~A}$ are almost identical. The wake voltage linearly increases with the beam current in this condition.

By adjusting the amplitude and phase of the rf voltage source, these wake voltages are canceled by the tube output currents as shown in the middle of Fig. 3. The suppressions of the driving harmonics are better than $30 \mathrm{~dB}$ for all cases. The remaining voltages seem to have the third harmonic component. The maximum normalized amplitude of $0.02 \mathrm{kV} / \mathrm{A}$ is observed with $I_{b}=20 \mathrm{~A}$. The waveforms of the rf voltage source normalized by $I_{b}$ are plotted in the bottom of Fig. 3. The normalized voltage
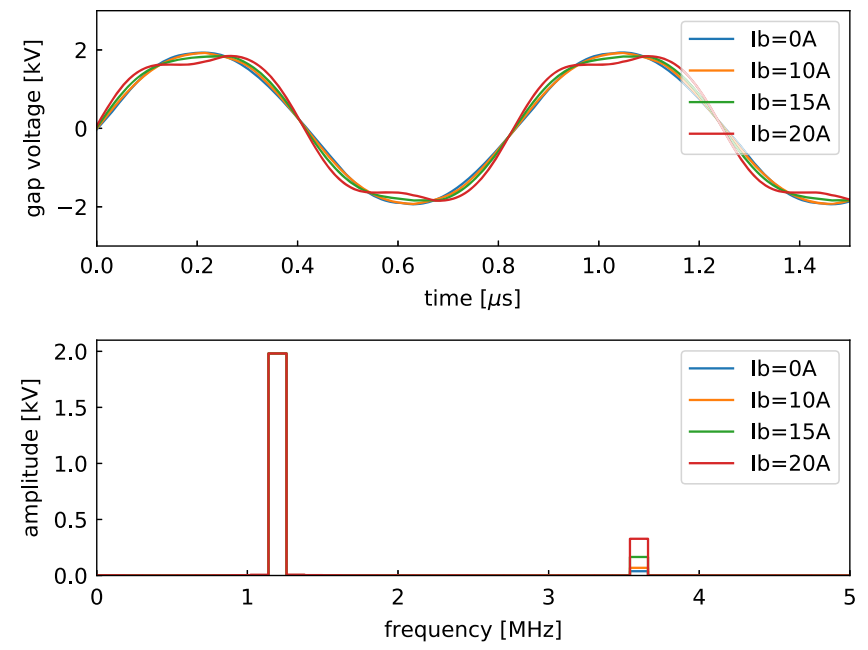

FIG. 4. (Top) Gap voltage waveforms with the compensation signal of the fundamental accelerating harmonic and (bottom) the frequency spectrum of the gap voltage.

decreases with the beam current; i.e., it shows nonlinear behavior. The nonlinearity comes mainly from the amplitude dependence of the tube gain. Also, the impedance of the tube seen by the beam varies when the tube generates the compensation current. Thus, beam loading compensation has a nonlinearity if the cavity is driven by vacuum tubes in a more nonlinear operation area.

Beam loading compensation with a driving rf voltage is also simulated. The amplitude and phase of the voltage source are adjusted so that the fundamental accelerating harmonic component of the gap voltage is $2 \mathrm{kV}$, for beam currents from 0 (no beam) to $20 \mathrm{~A}$. The beam phase that corresponds to the synchronous phase is set to 0 degrees. The simulated gap voltage waveforms and the frequency spectrum are plotted in Fig. 4. In the case without a beam, the voltage waveform has a slight distortion due to the third harmonic, which has the relative amplitude of a few percent to the fundamental accelerating harmonic, as shown in the bottom plot in the figure. When increasing the beam current, the amplitude of the third harmonic increases, while the fundamental accelerating harmonic is kept to $2 \mathrm{kV}$. With a beam current of $20 \mathrm{~A}$, the amplitude of the third harmonic reaches $0.32 \mathrm{kV}, 16 \%$ of the fundamental accelerating harmonic. The distortion of the gap voltage waveform becomes significant.

The required amplitude of the compensation signal is large in the case of high beam currents. Although the control grid is driven by the single harmonic rf signals, the high tube output current contains the higher harmonics, which cause the voltage waveform distortion in the wideband MA cavity.

The solid-state amplifier that is not included in the simulation is also a major source of nonlinearities. The dependency of the control grid voltage, which is driven by the solid-state amplifier, on the input of the driver 


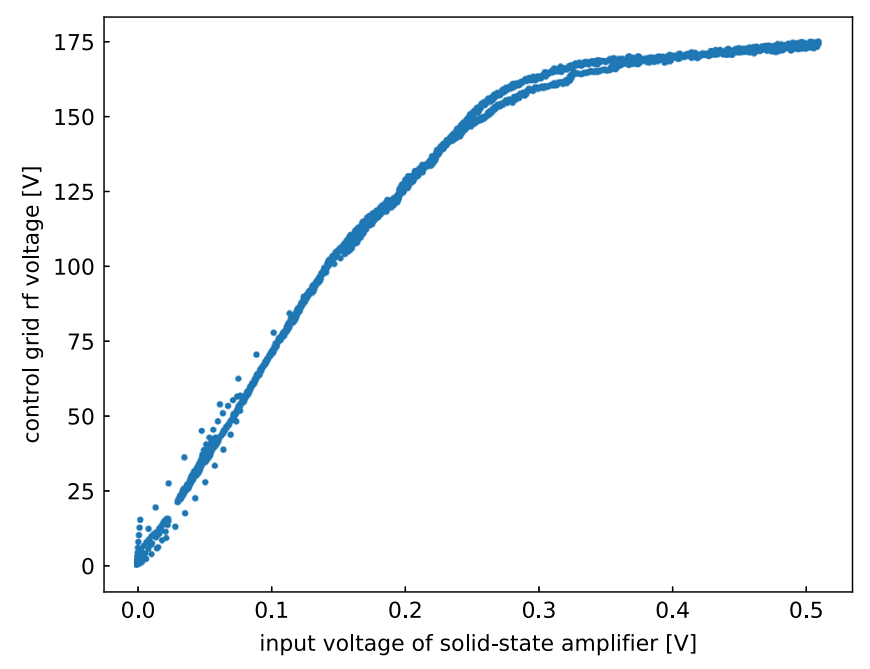

FIG. 5. Measured dependency of the control grid voltage on the input of the solid-state amplifier when accelerating the $1 \mathrm{MW}$ equivalent beam.

amplifier when accelerating the $1 \mathrm{MW}$ equivalent beam is plotted in Fig. 5. The signals contain several harmonics for multiharmonic beam loading compensation; therefore, the amplitudes of the fundamental accelerating harmonic $(h=2)$ are plotted. The input and control grid voltages vary during the accelerating period, where the cavity gap voltage varies according the voltage program and the condition of the beam loading also varies. For an input voltage of less than $0.25 \mathrm{~V}$, the control grid voltage, i.e., the output of the solid-state amplifier, increases linearly. One can clearly see a nonlinearity and saturation above the input voltage of $0.3 \mathrm{~V}$. This indicates that the existing solid-state amplifier is operated close to its limit when accelerating $1 \mathrm{MW}$ beams.

With these simulation and measurement results, the issues of the $\mathrm{rf}$ feedforward method are addressed. Nonlinearity of beam loading compensation implies that the optimum parameter of the feedforward is different for the various beam intensities and also the conditions of longitudinal painting, which changes the ratios of the harmonic components of the beam current.

The generation of the compensation signal causes the voltage waveform distortion in the wideband cavity due to the higher harmonic components of the tube output current. The compensation of the distortion is essentially difficult for the rf feedforward, which uses the beam signal to generate the compensation signals.

\section{MULTIHARMONIC VECTOR rf VOLTAGE CONTROL}

\section{A. System configuration}

The next-generation LLRF control system for the RCS based on MTCA.4 (micro telecom computing architecture [32]) is under development. The system overview is given in our previous article [33]. The capability of the MTCA.4 backplane for high-speed serial communication enables sophisticated signal transfer between the modules. Since available logic cells of the modern field programmable gate arrays (FPGAs) on the new modules are much more than that of old FPGAs in the existing LLRF control system, the number of modules needed to control 12 cavities is less than in the existing system. We classify the LLRF functions into two categories: the common function and the cavity driving function. The multiharmonic vector rf voltage control is implemented in the cavity driver module.

The multiharmonic vector if voltage control is designed so that it can control complex amplitudes of eight harmonics $(h=1 \ldots 8)$, while the existing system regulates scalar amplitudes of two harmonics $(h=2,4)$ [34]. The functional block diagram is shown in Fig. 6. The system clock frequency is $144 \mathrm{MHz}$.

The revolution frequency $(h=1)$ signal from the pattern memory in the common function module is serialized and distributed to the cavity driver modules via the backplane. The revolution frequency signal from the backplane is led to a phase accumulator. The phase accumulator generates the phase signal like a sawtooth wave varying from $-\pi$ to $\pi$ at the revolution frequency. By multiplying the selected harmonic number, higher harmonic phase signals can be generated, which are perfectly synchronized to the phase signal of the revolution harmonics.

Since the frequency is low compared to the system clock frequency, the cavity gap voltage signal is converted directly to digital signal by an analog-to-digital converter (ADC). The digitized signal is fed to the eight feedback blocks. The separating treatment of the several harmonics are commonly used for various purposes [35]. The feedback block has a classical $I / Q$ (in-phase-quadrature-phase) feedback structure. In the feedback blocks, the phase signal of $(h=1)$ is multiplied by the selected harmonic number $\left(h_{n}=1 \ldots 8\right)$. Cosine and sine signals of unity amplitudes for the $I / Q$ demodulation are generated by the coordinate rotation digital computer (CORDIC) block using the phase single of the selected harmonic. The $I / Q$ waveforms are recorded in a memory on the FPGA and used for commissioning and monitoring of the system.

The frequency response of the low-pass filter (LPF) determines the overall performance of the feedback loop. The configuration of the filter is described in following subsection.

A switch to open and close the feedback is implemented. Open loop measurements are important for commissioning.

The $I$ and $Q$ signals are compared with the set points from the $I / Q$ voltage pattern memory. The set points are updated every $1 \mu \mathrm{s}$. Through the proportional and integral (PI) controller, an rf signal is generated by the $I / Q$ modulation as 


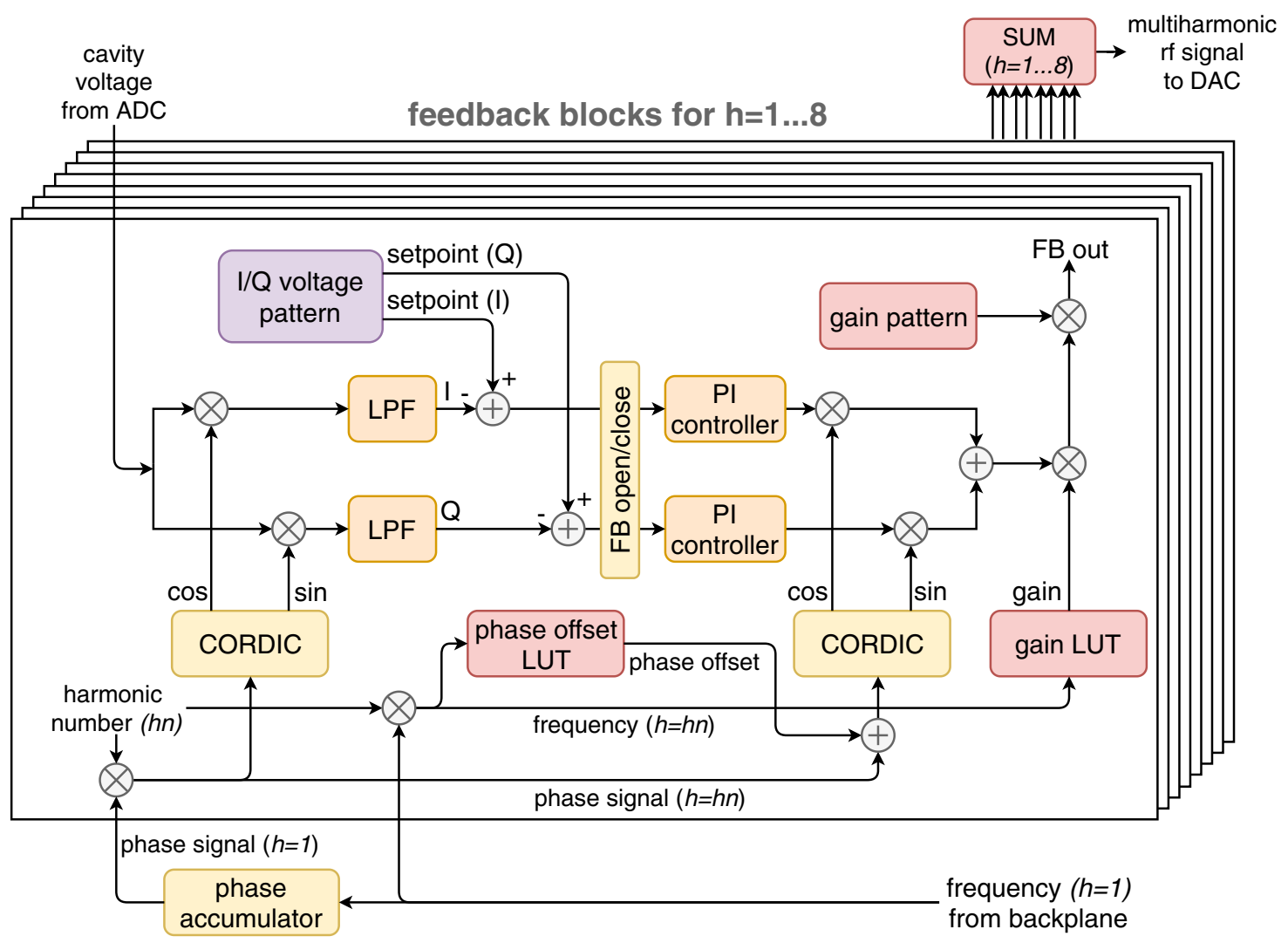

FIG. 6. Simplified block diagram of the multiharmonic vector rf voltage control.

$$
\begin{aligned}
(\mathrm{rf} \text { signal })= & I_{\mathrm{PI}} \cos \left(2 \pi h_{n} f_{\text {rev }} t+\phi_{\text {offset }}\right) \\
& +Q_{\mathrm{PI}} \sin \left(2 \pi h_{n} f_{\text {rev }} t+\phi_{\text {offset }}\right),
\end{aligned}
$$

where $I_{\mathrm{PI}}$ and $Q_{\mathrm{PI}}$ are the output $I / Q$ signals of the PI controllers and $f_{\text {rev }}$ is the revolution frequency. The phase offset look-up table (LUT) gives the phase offset $\phi_{\text {offset }}$ between the $I / Q$ demodulator and modulator to adjust the phase of the transfer function of the system. For addressing of the LUT, the digital frequency signal of the selected harmonic, $h_{n} \times f_{\text {rev }}$, is used. The bit width of the frequency signal is 32 bit and 14 bit of it is picked up for addressing. The gain LUT is implemented to compensate the amplitude response of the cavity and the amplifier chain, which consists of the solid-state amplifier as a driver and the final stage vacuum tube amplifier. These LUTs are necessary to cover the wide frequency sweeps of the harmonics from the revolution harmonics $(h=1)$ to the higher harmonics $(h=8)$ during acceleration.

By applying the gain pattern for changing the system gain along the time during the cycle, the output of the feedback block is generated.

Finally, the multiharmonic of signal is obtained by summing up the signals from the eight feedback blocks. The signal is converted to an analog rf signal by a digital-toanalog converter (DAC), and it is sent to the amplifier chain to drive the cavity.

\section{B. Configuration of the low-pass filer}

The role of the LPF in the $I / Q$ demodulator is to remove the spectrum of the other harmonics than the selected harmonics, as well as to reject the noise outside the bandwidth. In the case of the J-PARC RCS, the frequency distance of the harmonics varies as the revolution frequency changes from 614 to $835 \mathrm{kHz}$. For amplitude control with less than $1 \%$ accuracy, the LPF should reject the other harmonics better than $60 \mathrm{~dB}$. The bandwidth of the LPF should be a few hundred kilohertz, which is very narrow compared to the system clock frequency of $144 \mathrm{MHz}$.

The amplitude and phase responses of the LPF affect the performance of the feedback loop, such as the feedback gain, the bandwidth, and the stability. One should carefully consider the configuration of the LPF.

In the existing LLRF control system, the 63-tap finite impulse response (FIR) filter is employed as the LPF [34] to achieve narrow band filtering. We avoid employing the FIR filter, which requires many multipliers in its configuration, for implementation of the multiharmonic vector voltage control for eight harmonics. Although modern FPGAs offer a lot of logic cells, the number of multipliers is still limited. We explore economic solutions in terms of FPGA logic for the LPF configuration.

The cascaded integrator-comb (CIC) filter [36] is widely used in signal processing as a low-pass filter. It is economically implemented without multipliers. Although it has a 

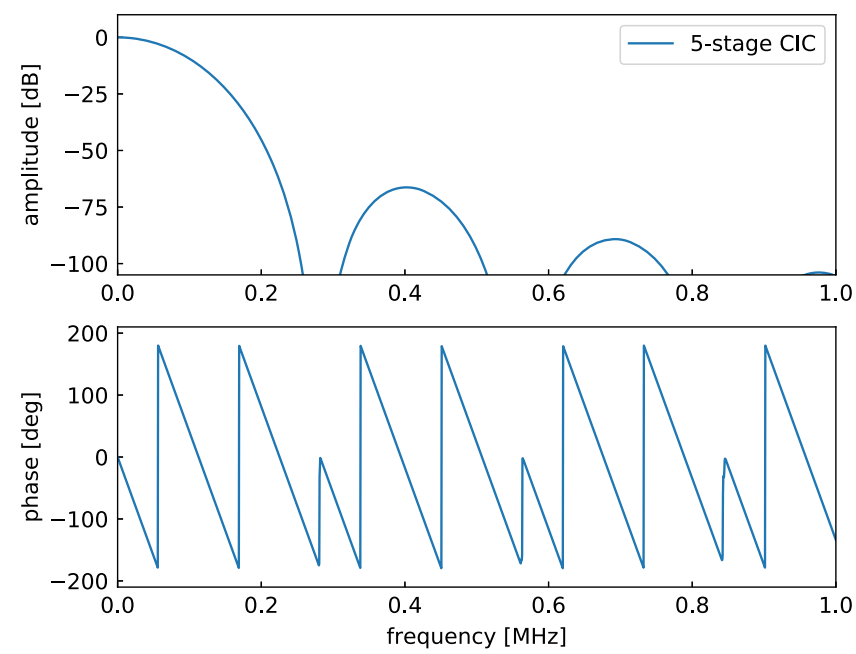

FIG. 7. Amplitude and phase responses of the five-stage CIC filter where $R=2, M=256$, and $N=5$.

feedback path in the integrator part, the response is identical to a moving average FIR filter, and the phase response is linear.

To treat the digital filters, the $z$ transform is used. The transfer function of a CIC filter $H_{\mathrm{CIC}}$ on the $z$ plane is expressed as

$$
H_{\mathrm{CIC}}(z)=\left(\frac{1-z^{-R M}}{1-z^{-1}}\right)^{N}
$$

where $R$ is the decimation factor between the integrator running at the full system clock frequency $f_{\text {clk }}$ and the comb running at the lower frequency at $f_{\text {clk }} / R, M$ the number of delay taps in the comb, and $N$ the number of stages.

To realize narrow band filtering, the CIC filter with $R=2$, $M=256$, and $N=5$ is implemented for our prototype of the multiharmonic vector rf voltage control [37]. The amplitude and phase responses of the five-stage CIC are plotted in Fig. 7. As shown in the figure, the rejection for the frequency range of the neighbor harmonics, from 600 to $850 \mathrm{kHz}$, is sufficient better than $80 \mathrm{~dB}$. On the other hand, the phase rotation in the passband is large due to cascading five stages, which implies that with the five-stage CIC filter the proportional gain in the PI controller is limited for stability of the feedback.

The novel tracking CIC filter proposed by Molendijk [38] is a CIC filter with notches which follow the change of the revolution frequency, where the system clock is fixed. The filter structure was developed for application in the CERN PS booster.

The simplified block diagram of the tracking CIC filter is illustrated in Fig. 8. The revolution frequency is multiplied by 32 , and the phase accumulator generates the phase signal of $(h=32)$. The clock enable (CE) pulse is generated by using the zero crossing of the phase signal.
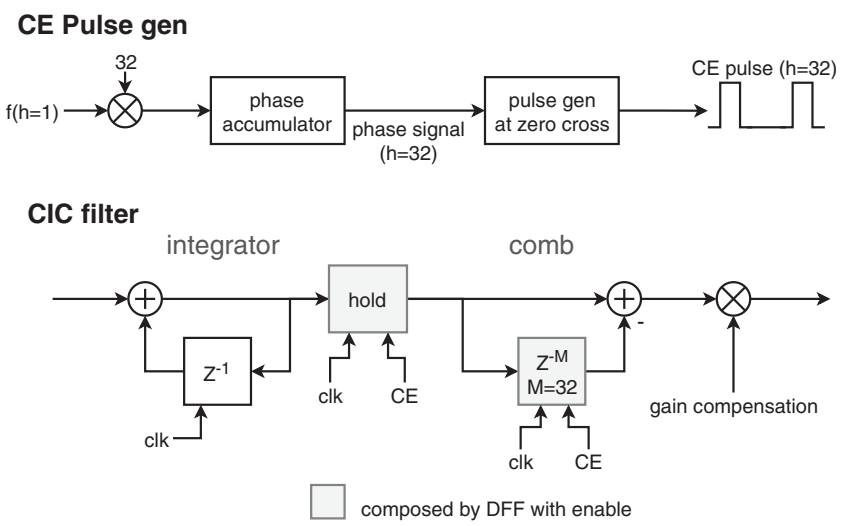

FIG. 8. Simplified block diagram of the tracking CIC filter.

Therefore, the CE pulse at the frequency of $(h=32)$ is generated.

In the CIC filter, the integrator part runs at the system clock frequency as the normal CIC filters. The delay in the comb part is driven by the CE pulse, where the hold and delay is implemented by D-flip-flops (DFFs). By setting $M=32$, the notches at the harmonics of the revolution harmonic are realized. The overall gain of the filter is proportional to $f_{\text {rev }}$; therefore, the compensation gain $G_{\text {comp }}$ is applied as

$$
G_{\text {comp }} \propto f_{\text {rev }}
$$

so that the overall gain is constant.

The basic idea behind the tracking CIC filter and our filter with smoothly varying coefficients [39] is essentially similar in terms of changing the delay taps in the filter. Since a much faster system clock is allowed in the modern FPGA, the resolution of the delay is more precise now; the implementation of the tracking CIC filter is much more simple than our previous filter.

The amplitude and phase responses of the tracking CIC filter for $f_{\text {rev }}=600$ and $850 \mathrm{kHz}$ are plotted in Fig. 9 . The notches follow the change of $f_{\text {rev }}$. Here, one should notice that the $\mathrm{CE}$ pulse period is quantized and fluctuates within one system clock period. The frequency of the notch therefore fluctuates, while the average in time is close to $f_{\text {rev }}$. This may affect the rejection of the neighbor harmonics. The effect is more important for higher $f_{\text {rev }}$, i.e., for the smaller number of delay clocks in the comb part. For the J-PARC RCS, the maximum $f_{\text {rev }}$ is $850 \mathrm{kHz}$, and the system clock frequency $f_{\text {clk }}$ is $144 \mathrm{MHz}$. The delay in the comb part is obtained by using the ratio as

$$
\frac{f_{\mathrm{clk}}}{f_{\text {rev }}}=169.41 \ldots ;
$$

thus, the delay in the comb part alternates between 169 and 170 clocks. The amplitude responses around the first notches near $f_{\text {rev }}$ with a delay of 169 and 170 clocks are plotted in Fig. 10. For both delays of 169 and 170, the attenuations at $850 \mathrm{kHz}$ are on the order of $50 \mathrm{~dB}$, worse 

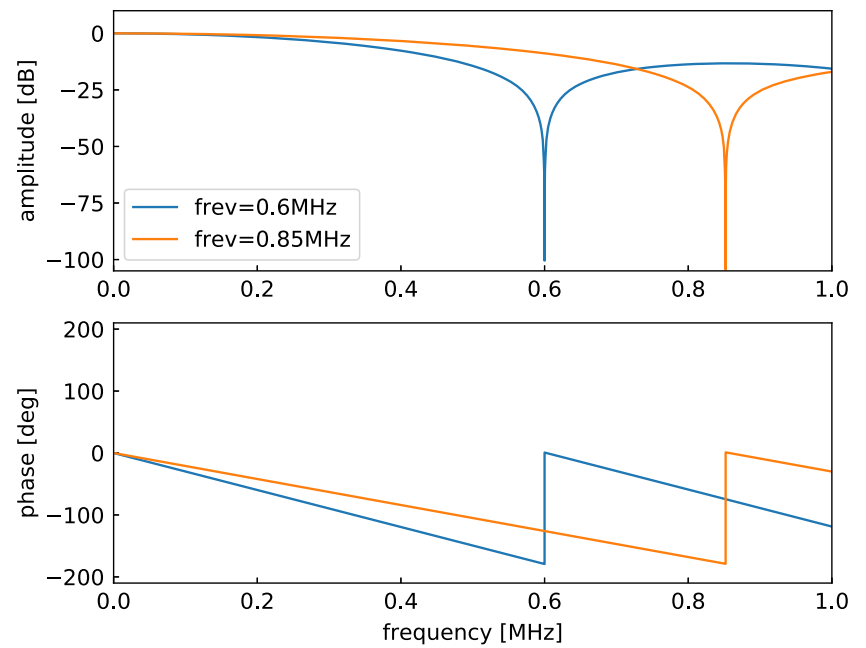

FIG. 9. Amplitude and phase responses of the tracking CIC filter for $f_{\text {rev }}=600$ and $850 \mathrm{kHz}$.

than our target value of $60 \mathrm{~dB}$. Adding a LPF or simply cascading the tracing $\mathrm{CIC}$ is required to achieve an attenuation of better than $60 \mathrm{~dB}$.

While the tracking CIC filter has a nice feature to follow the change of the revolution frequency, the bandwidth is too wide. The bandwidth is wider than the possible feedback bandwidth limited by the long delay of the cable between the LLRF control room to the tunnel on the order of $2 \mu \mathrm{s}$ round trip.

By these reasons above, the combination of the tracking CIC filter and leaky integrator (LI), which is called a "hybrid filter" in this article, is considered. The block diagram of the leaky integrator is illustrated in Fig. 11. The leaky integrator can be implemented economically with a coefficient of $2^{k}$, where the multiplier can be replaced with bit shift.

The transfer function of the leaky integrator $H_{\mathrm{LI}}$ is

$$
H_{\mathrm{LI}}(z)=\frac{z^{-1}}{2^{k}+\left(1-2^{k}\right) z^{-1}} .
$$

The amplitude and phase responses of the leaky integrator are plotted in Fig. 12. With increasing $k$, the bandwidth becomes narrower, and the attenuation also increases. It has a nonlinear phase response. The phase quickly reaches $90^{\circ}$

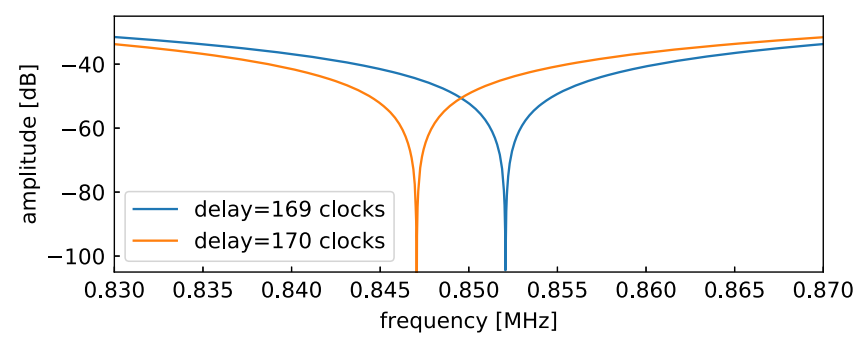

FIG. 10. Amplitude response of the tracking CIC filter for $f_{\text {rev }}=850 \mathrm{kHz}$, with a delay of 169 and 170 clocks.

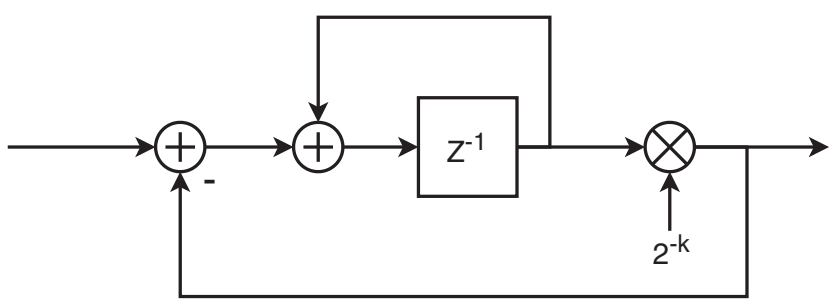

FIG. 11. Simplified block diagram of the leaky integrator with a coefficient of $2^{k}$.
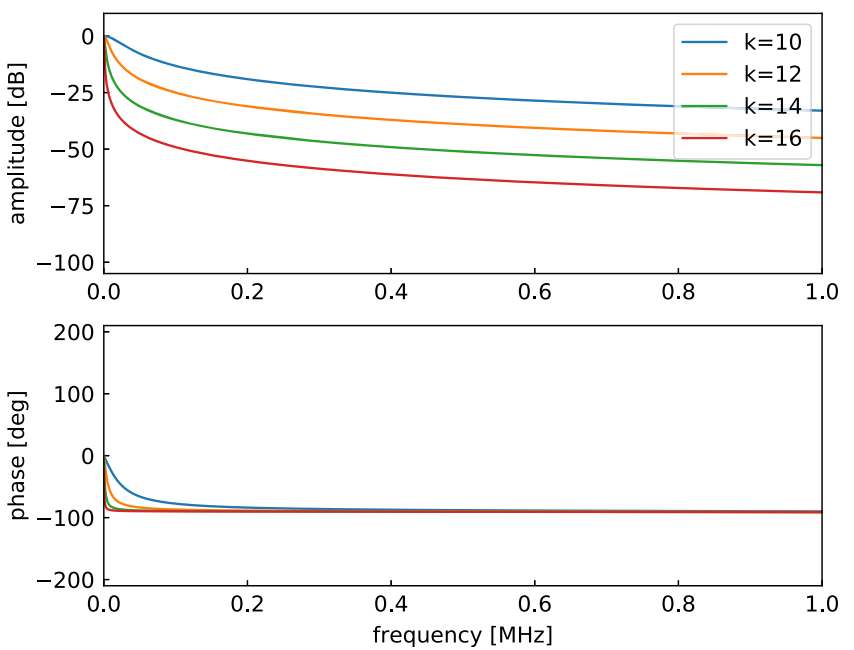

FIG. 12. Amplitude and phase responses of the leaky integrator for $k=10,12,14,16$.

and stays there for higher frequencies of more than $200 \mathrm{kHz}$. The leaky integrator alone does not meet our requirements. With small $k$, the attenuation at the revolution harmonics is not sufficient. An attenuation of more than $60 \mathrm{~dB}$ is possible with $k=16$; however, the bandwidth is too narrow, and a very limited feedback bandwidth is expected.

The amplitude and phase responses of the hybrid filter, which is composed by cascading the tracking CIC filter and the leaky integrator, are plotted in Fig. 13. A value of $k=10$ in the leaky integrator is chosen. The attenuations at the revolution frequencies are satisfactorily better than $60 \mathrm{~dB}$. The bandwidth is moderate, and the phase shift in the passband is not too much.

The hybrid filter, which satisfies our requirement and still is realized economically in terms of FPGA logic, is used for the beam tests.

\section{COMMISSIONING AND CHARACTERIZATION OF THE SYSTEM}

\section{A. Commissioning of the LUTs}

Contrary to the rf feedforward, the commissioning of the feedback does not require proton beams. The phase offset 

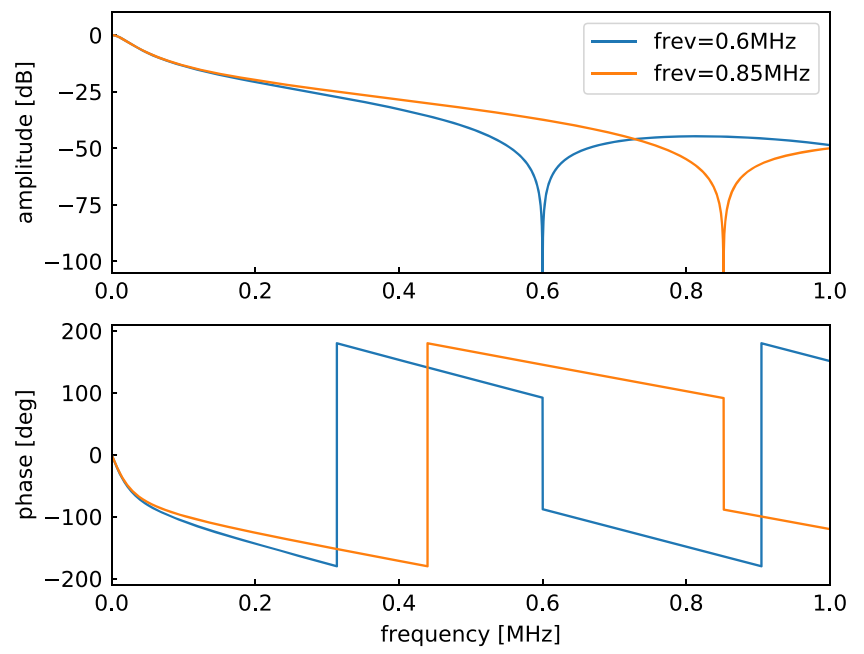

FIG. 13. Amplitude and phase responses of the hybrid filter for $f_{\text {rev }}=600$ and $850 \mathrm{kHz}$. A value of $k=10$ is chosen in the leaky integrator.

and gain LUTs must be set properly to cover the wide frequency range.

Figure 14 shows the setup of the commissioning of the feedback without beams. The output signal of the system is sent to the device under test (DUT), which consists of the amplifier chain and the cavity. The cavity gap monitor signal is led to the input of the system. For commissioning of the LUTs, the five-stage CIC filter is used.

The phase offset LUT is most important to close the feedback loop. Since the DUT includes many components, it is difficult to predict the frequency response of the DUT. The LUT is adjusted based on the following procedure.

An open loop measurement is performed with the condition that the $I / Q$ set point is set as $I=1000, Q=0$ (digital values) and the phase LUT is set to zero for all frequencies. The gain LUT is set to unity. The $I / Q$ amplitudes of the cavity voltage are measured at 200 points for the frequency range from 0.4 to $8.99 \mathrm{MHz}$.

The measured $I$ and $Q$ at the 200 points are plotted in the top plot in Fig. 15. In the figure, the circles represent the measured data, and the lines are the interpolated ones.

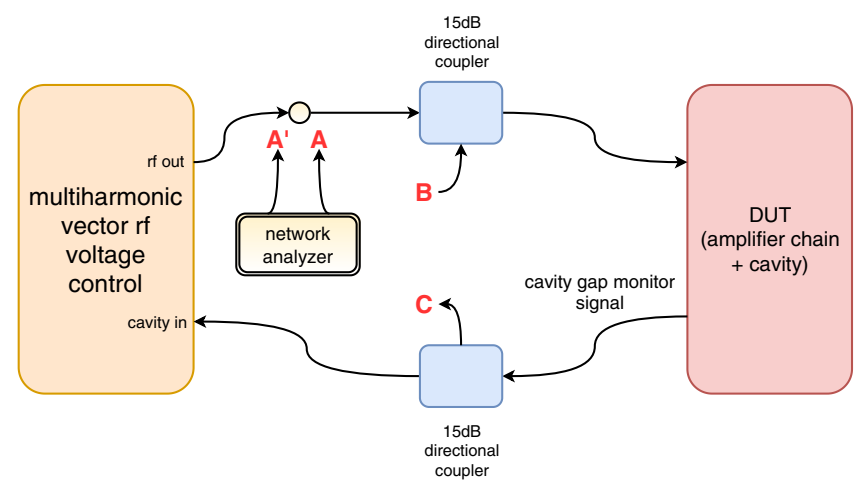

FIG. 14. Commissioning setup.

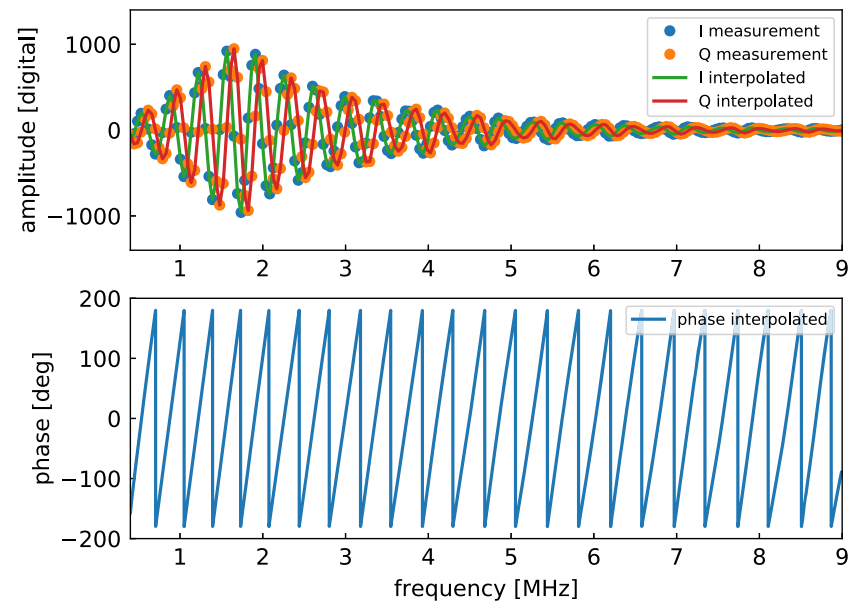

FIG. 15. (Top) The measured $I$ and $Q$ at the 200 points and the interpolated ones and (bottom) the phase response calculated from the interpolated $I$ and $Q$, when the phase offset LUT is set to zero for all frequencies.

One can see that the $I$ and $Q$ oscillate; therefore, the phase response of the DUT has to be taken into account. From the interpolated data, the phase response plotted in the bottom plot in Fig. 15 is calculated as

$$
\text { (phase) }=\arg (I+i Q) .
$$

The almost linear phase response indicates that the main source of the response is a delay, which consists of the cable delay and the digital delay in the system. The phase response is also due to the cavity resonator and the electrical circuits of the amplifier chain, while the effects are relatively small.

By using the measured phase response, the phase offset LUT is set. The measured $I, Q$, and the phase response with the LUT set are plotted in Fig. 16. Clearly, the phase response of the DUT is compensated by the LUT, while small errors are observed in the higher-frequency region because of small amplitudes. In this condition, if the set point to drive the system has the $I$ amplitude only as $\left(I_{\text {set }}, 0\right)$, the received signal from the gap monitor contains the $I$ amplitude only as $\left(I_{\text {recv }}, 0\right)$, and vice versa. This is the condition to close the feedback loop. The feedback loops for $I$ and $Q$ are separated, and each loop can be handled as a simple single-input-single-output (SISO) system.

The amplitude response of the $I$ value shown in Fig. 16 is mainly caused by the cavity response and the low-pass characteristics of the input circuit of the vacuum tube. This implies that the feedback gain is reduced especially for the high-frequency region higher than $(h=6)$. The amplitude response of the DUT is compensated by the gain LUT.

From the interpolated $I / Q$ data shown in Fig. 16, the gain LUT is set so that the signal amplitude

$$
(\text { amplitude })=\sqrt{I^{2}+Q^{2}}
$$




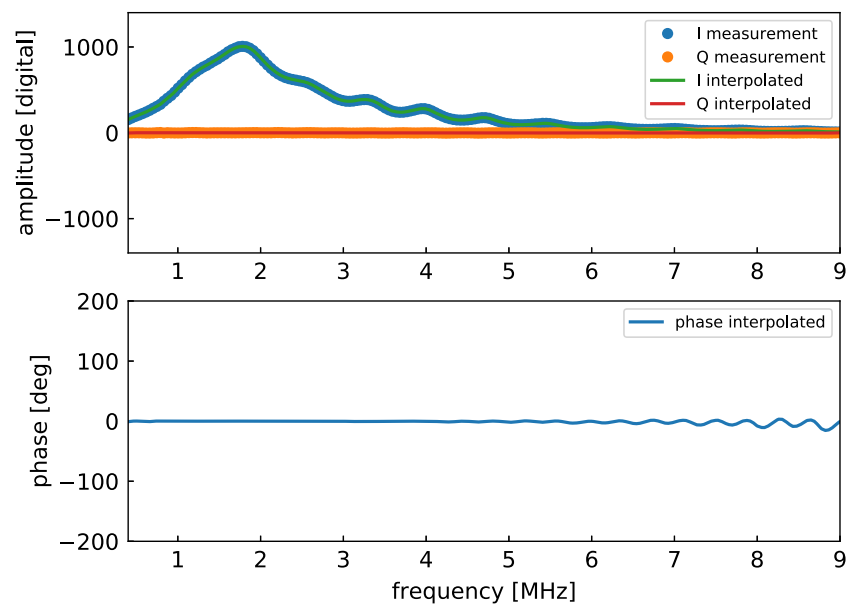

FIG. 16. (Top) The measured $I$ and $Q$ at the 200 points and the interpolated ones and (bottom) the phase response calculated from the interpolated $I$ and $Q$, when the phase offset LUT is set by using measured $I$ and $Q$.

is constant. The amplitude at the cavity resonance frequency $(1.7 \mathrm{MHz})$ is chosen as the target amplitude. We limit the maximum gain to a factor of 10 to avoid the output saturation of the system.

Because of the nonlinearity of the system due to the vacuum tube, we need iterations of the LUT setting. The measured amplitude responses with the constant gain LUT, the first and second gain LUT setting, are compared in Fig. 17. With the first LUT setting (gain LUT set 1), overcompensation is observed. By iteration (gain LUT set 2), an almost flat amplitude response is obtained up to $5.5 \mathrm{MHz}$. Above the frequency, the maximum gain of 10 is applied; therefore, the compensation is not sufficient and decay is observed. With the gain LUT set, the feedback gain for the harmonics up to $(h=6)$ is expected to be flat. The gain is reduced for $(h=7,8)$; however, still 10 times

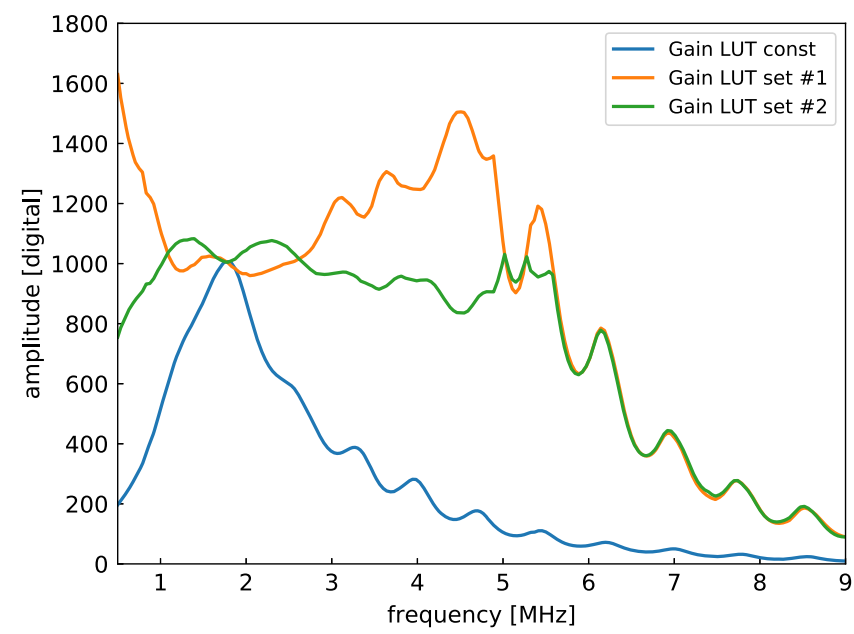

FIG. 17. Comparison of the amplitude responses with the constant gain LUT, the first and second gain LUT setting.

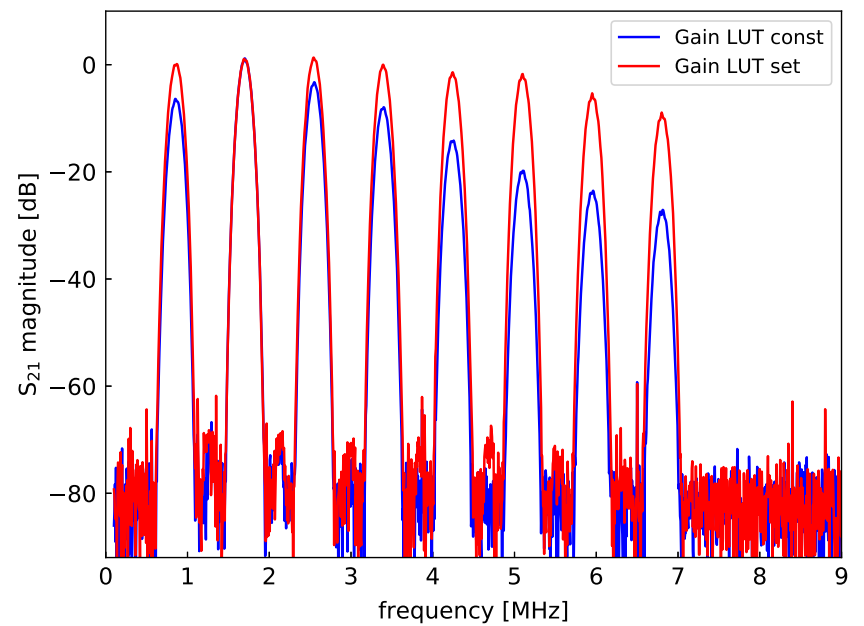

FIG. 18. Magnitudes of multiharmonic open loop transfer functions in the cases of the gain LUT const and set. The five-stage CIC filter is used in the feedback blocks for the measurement.

higher gain is achieved than in the case of the gain LUT constant.

The flat gain response is obtained at a moderate amplitude of the driving signal. Since the tube gain may vary with a higher amplitude of the driving signal when accelerating high-intensity beams as shown in Sec. II, the PI gain setting should be conservative to keep the stability margin of the feedback.

A multiharmonic open loop measurement is performed to see the effect of the gain LUT set. For the measurement, the five-stage CIC filter is used, and the revolution frequency $(h=1)$ is set to $0.85 \mathrm{MHz}$. The $I / Q$ set point is $(0,0)$, and the feedback switch is closed. The feedback blocks for all eight harmonics are enabled. The connection between $A$ and $A^{\prime}$ in Fig. 14 is opened so that the transfer function $S_{21}$ from $A$ to $A^{\prime}$ is measured by a network analyzer.

The measured magnitudes of $S_{21}$ in the cases of the gain LUT constant and gain LUT set are compared in Fig. 18. The bandwidth of the five-stage CIC filter is narrow enough, and the passband of each harmonic is clearly separated from the others. The gains of the higher harmonics $(h=6,7,8)$ are smaller than $-20 \mathrm{~dB}$ compared to the fundamental accelerating harmonic $(h=2)$, which is near the resonance. With the gain LUT set, the gains of that harmonic are increased. The gains are fairly aligned for harmonics up to $(h=6)$, while harmonics $(h=7,8)$ have lower but improved gains.

Thus, the methodology to adjust the phase offset and gain LUTs for covering the wide frequency range is established.

\section{B. Characterization of the feedback}

By setting up the phase offset LUT, the condition to close the feedback is satisfied. The transfer function $S_{21}$ from point $B$ to $C$ is measured as the closed loop gain of the feedback 

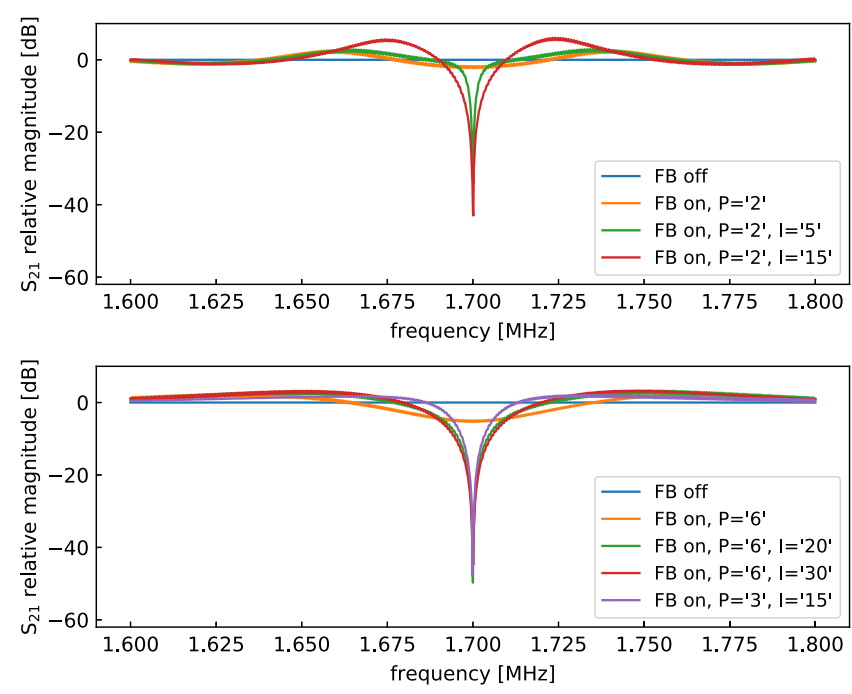

FIG. 19. Normalized closed loop gains with various PI gain settings at $1.7 \mathrm{MHz}$, in the cases of (top) the five-stage CIC filter and (bottom) the hybrid filter.

loop, where the $I / Q$ set point is $(0,0)$ and the feedback switch is closed. Here, the rf signal from the network analyzer is regarded as the disturbance into the feedback.

The normalized closed loop transfer functions measured by the network analyzer with various proportional and integral gain settings around the fundamental accelerating harmonic $(h=2)$, where the revolution frequency $(h=1)$ is set to $0.85 \mathrm{MHz}$, in the cases of the five-stage CIC filter and the combination of the tracking CIC and LI, are plotted in Fig. 19. $P$ and $I$ are the 8-bit digital values of the proportional and integral gains, respectively. The transfer function $H_{\mathrm{PI}}(z)$ of the PI controller in the feedback block is expressed as

$$
H_{\mathrm{PI}}(z)=K_{P}+K_{I} \frac{1}{1-z^{-1}},
$$

where $K_{P}$ and $K_{I}$ are the proportional and integral gains, respectively. In the feedback block, the relations between the digital value and the actual value are $K_{P}=\mathrm{P} / 8$ and $K_{I}=\mathrm{I} / 36864$, respectively.

In both cases with the five-stage CIC filter and the hybrid filter, the phase shifts in the passband due to the system delay and filter configuration limit the proportional gain. Typically, we can put 2 and 6 as $P$ for the five-stage CIC and hybrid filter, respectively. The effects of the small proportional gains on the closed loop gain are insufficient. However, by applying the integral gain, the closed loop gain, i.e., the reduction of the cavity impedance, is better than $40 \mathrm{~dB}$ in both cases. In general, a higher gain is possible and a wider bandwidth is achieved with the hybrid filter than the five-stage CIC filter, which has more phase shift in the passband. With $P=2$ and $I=15$ for the fivestage CIC filter, a comparable bandwidth can be achieved, while unwanted enhancement of the disturbance of more than $6 \mathrm{~dB}$ is observed at 1.675 and $1.725 \mathrm{MHz}$, which is not

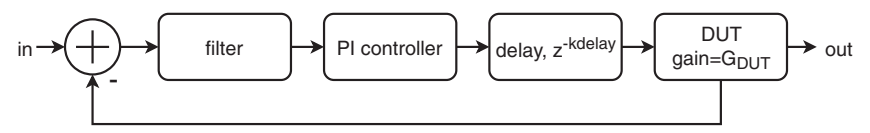

FIG. 20. The feedback model in the baseband.

preferred. The measurements show the advantage of the hybrid filter over the five-stage CIC filter.

For the beam operation, relatively conservative gains, $P=3$ and $I=15$, are used to keep stability margins for gain variation of the tube amplifier. The loop gain with the PI gains is still $7.7 \mathrm{~dB}$ at $4 \mathrm{kHz}$ away from the center frequency, where $4 \mathrm{kHz}$ is the maximum synchrotron frequency of the RCS during the acceleration cycle.

The baseband simulation [40] is useful to characterize the feedback. The feedback model in the baseband is shown in Fig. 20. As described in the previous section, the feedback is treated as a SISO system, and the DUT can be regarded as a gain with the proper setting of the phase offset LUT. The free parameters in the system are the delay and the DUT gain $G_{\text {DUT }}$. The number of delay clocks $k_{\text {delay }}$ is the integer part of $\frac{T_{\text {delay }}}{T_{\text {clk }}}$, where $T_{\text {delay }}$ is the total delay and $T_{\text {clk }}$ is the clock period. The transfer function of the delay $H_{\text {delay }}$ is

$$
H_{\text {delay }}(z)=z^{-k_{\text {delay }}} .
$$

The open loop transfer function is

$$
H_{\text {filter }} \cdot H_{\text {PI }} \cdot H_{\text {delay }} \cdot G_{\text {DUT }} \text {, }
$$

where $H_{\text {filter }}$ is the transfer function of the selected filter configuration. The closed loop gain is calculated by using the open loop transfer function.

The delay and gain are determined by using the open loop measurement from $A$ to $A^{\prime}$ in Fig. 14 with the PI gains of $K_{P}=1$ and $K_{I}=0$. The top of Fig. 21 shows the comparison of the measured and the simulated open loop transfer functions where $T_{\text {delay }}=3.1 \mu \mathrm{s}$ and $G_{\text {DUT }}=1.1$. In the plots, the horizontal axis is the frequency difference from the center frequency of the selected harmonic. The delay of $3.1 \mu \mathrm{s}$ consists of the inevitable cable delay of $2 \mu \mathrm{s}$ and the digital circuit delay of $1.1 \mu \mathrm{s}$. A major part of the digital circuit delay, about $700 \mathrm{~ns}$, is due to the serial connection between the ADC and FPGA. As a compromise, we will leave it as is at this moment. The simulation agrees well with the measurement in the low-frequency region below $0.4 \mathrm{MHz}$, but deviations for the magnitude and phase are seen above $0.4 \mathrm{MHz}$. This is because the simulation does not take the response of the cavity resonator into account. Since we are interested in the very-low-frequency region below $0.1 \mathrm{MHz}$ for the narrow band feedback, the deviations are not problematic.

Using the parameters $T_{\text {delay }}$ and $G_{\text {DUT }}$, the open and closed loop transfer functions are calculated with $P=3$ and $I=15$ (digital values) as plotted in the middle of Fig. 21. Also, the closed loop gain is compared to the measurement. Note that the open loop gain with the integral 

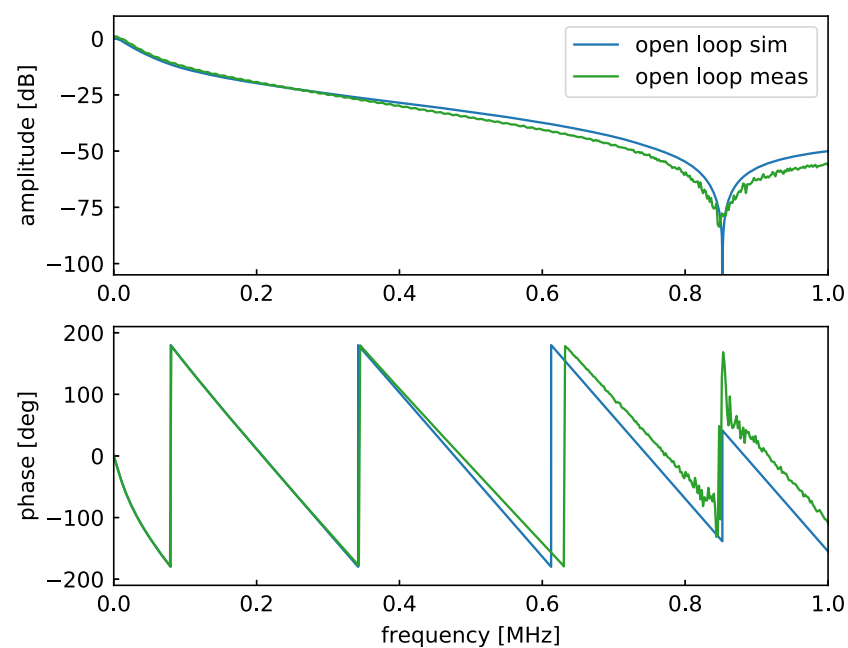

(a)
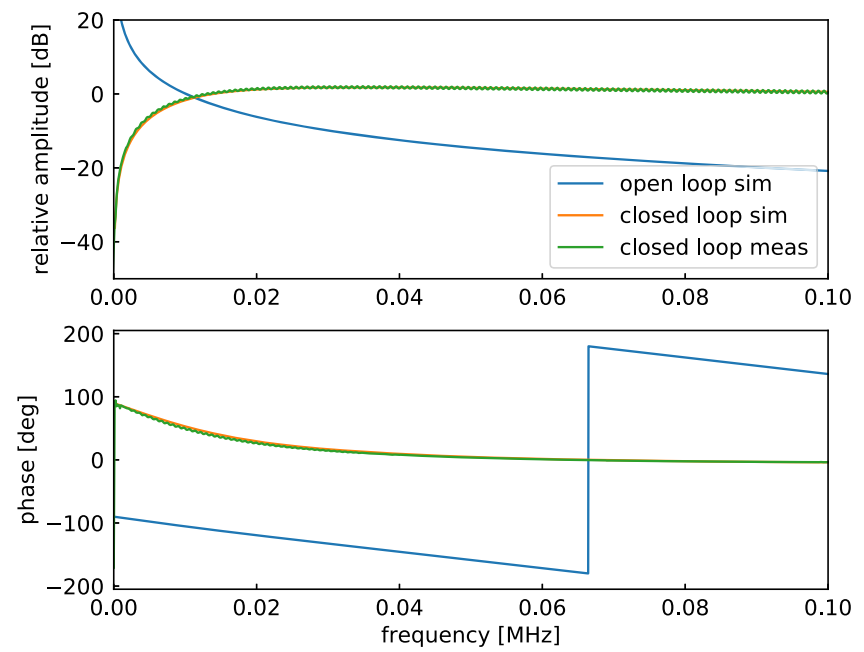

(b)

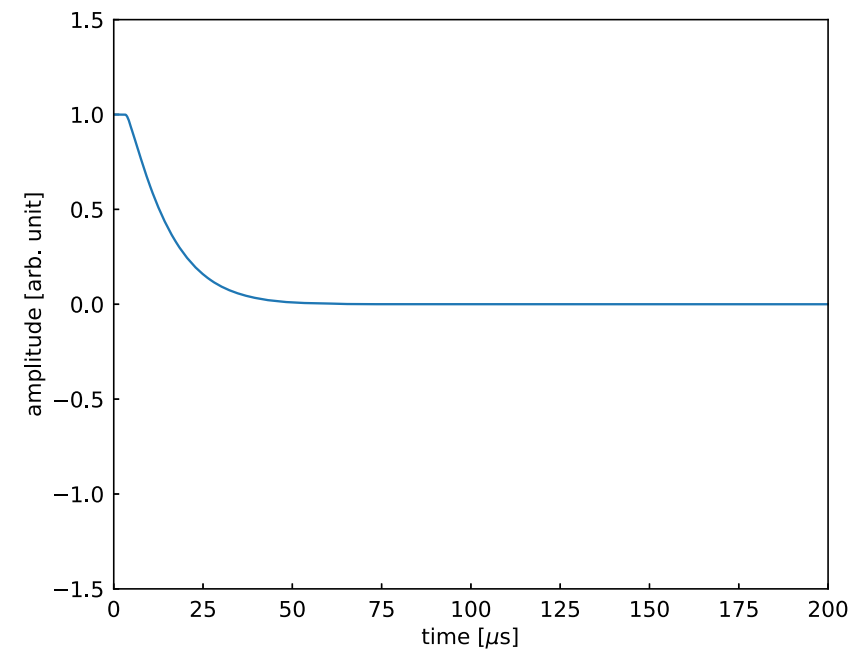

(c)

FIG. 21. (Top) Comparison of the measured and simulated open loop transfer functions where $T_{\text {delay }}=3.1 \mu \mathrm{s}$ and $G_{\text {DUT }}=1.1$. (Middle) Simulated open and closed loop transfer function with the PI gains and the measured closed loop transfer function. (Bottom) Simulated step response with the PI gains, $P=3$ and $I=15$.
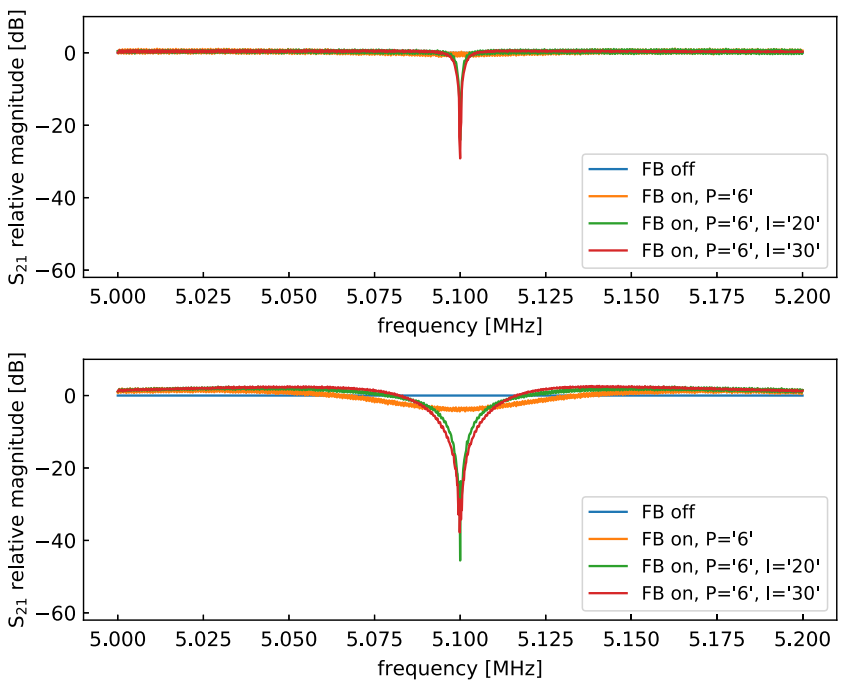

FIG. 22. Normalized closed loop gains with various PI gain settings at $5.1 \mathrm{MHz}$, in the cases of (top) the constant gain LUT and (bottom) the gain LUT set.

gain is difficult to measure, since the integrator may overflow if the loop is open. From the simulated open loop gain, the gain and phase margins are estimated as $17.1 \mathrm{~dB}$ and $85^{\circ}$ at the phase and gain crossover frequencies of $66.1 \mathrm{kHz}$ and $10.1 \mathrm{kHz}$, respectively. One can see that the closed loop simulation reproduces the measurement very well in terms of both the magnitude and phase.

The step response is also predicted by the simulation setup as plotted in the bottom of Fig. 21. The response with the PI gains reasonably settles down to zero within $50 \mu \mathrm{s}$.

Similar simulation results in the case of the five-stage CIC filter are reported in our previous article (Figs. 6 and 7 in Ref. [40]). The feedback bandwidth $P=2$ and $I=5$ (digital values), where the five-stage CIC filter and the PI gains are used in the beam tests with our prototype module [37], is narrower. The simulated step response is slow, and it takes more than $150 \mu$ s to reach zero.

These simulation results also show the advantage of the hybrid filter over the five-stage CIC filter.

The normalized closed loop transfer functions measured by the network analyzer with various proportional and integral gain settings around the third $(h=6,5.1 \mathrm{MHz})$ and fourth $(h=8,6.8 \mathrm{MHz})$ harmonics of the accelerating harmonic $(h=2)$ with the gain LUT constant and set are plotted in Figs. 22 and 23, respectively. With the gain LUT constant, the feedback bandwidths are much narrower than the fundamental accelerating harmonic $(h=2)$ near the cavity resonance frequency shown in the bottom of Fig. 19, while feedback gains better than $30 \mathrm{~dB}$ at the center frequencies are achieved thanks to the integral gains. For the third harmonic $(h=6)$, the response is fairly close to that of the accelerating harmonic $(h=2)$ with the gain LUT set. In the case of the fourth harmonic $(h=8)$, the bandwidth is still narrower than $(h=2)$, since the limited gain of 10 is 

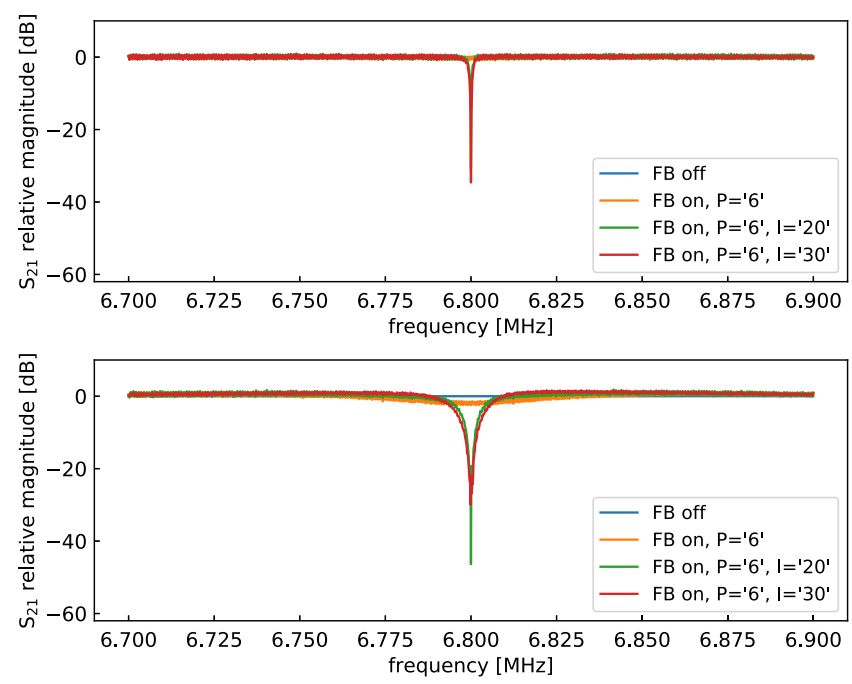

FIG. 23. Normalized closed loop gains with various PI gain settings at $6.8 \mathrm{MHz}$, in the cases of (top) the constant gain LUT and (bottom) the gain LUT set.

applied at that frequency. These results are consistent with the open loop measurement shown in Fig. 18.

Thus, setting up the gain LUT is important for multiharmonic beam loading compensation.

\section{MULTIHARMONIC rf VOLTAGE REGULATION}

\section{A. Reduction of voltage waveform distortion}

Voltage waveform distortion is an issue with the combination of the vacuum tube amplifier and the wideband MA cavity. The output current of the vacuum tube contains not only the driven harmonic but also higher harmonics, which generate the higher harmonic voltages. Since we employ a push-pull configuration for the final stage amplifier, the second harmonic voltage is fairly suppressed. However, the third harmonic voltage becomes non-negligible especially when the output current is high, i.e., the beam loading is heavy.

The distortion can be suppressed by the multiharmonic vector rf voltage control, while it essentially cannot be compensated by the rf feedforward using the beam current signals.

The performance of the control for reduction of the distortion is examined. In the top plot in Fig. 24, a distorted waveform is shown. The driven harmonic is chosen $(h=1)$ at $600 \mathrm{kHz}$ for the test to enhance the distortion to be compensated, while the $(h=2)$ is the driven harmonic for the normal operation. The feedback for the driven harmonic $(h=1)$ is closed, and the other harmonics $(h=2 \ldots 8)$ are open. The harmonic components of the distorted waveform are plotted in the top plot in Fig. 25. The programed voltage pattern has a trapezoidal shape, which has a rise and fall time of $0.1 \mathrm{~ms}$ and a flattop of $2 \mathrm{~ms}$. The driven harmonics is well regulated at the amplitude of $2 \mathrm{kV}$. The third
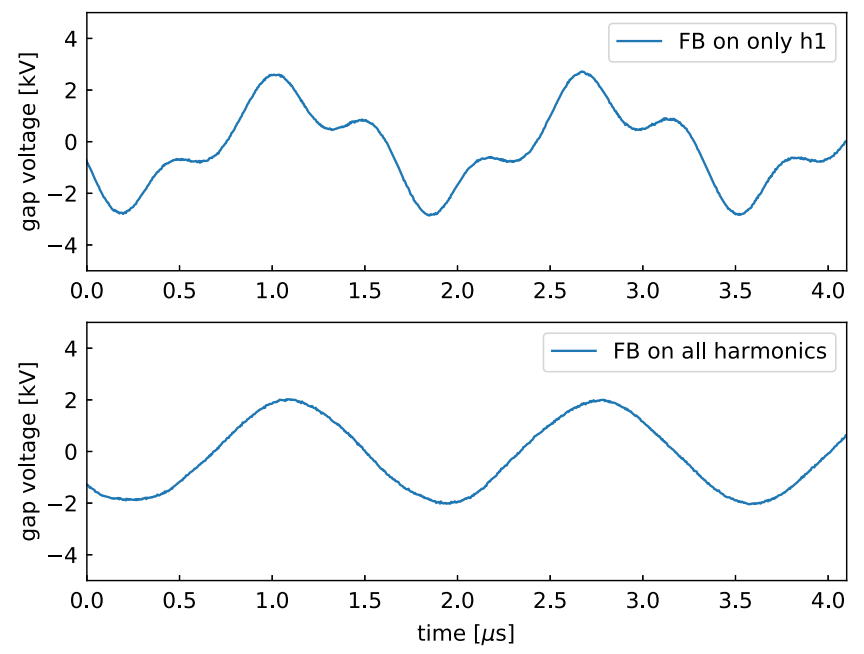

FIG. 24. (Top) Distorted gap voltage waveform when only the feedback for $(h=1)$ is closed and the other harmonics ( $h=2 \ldots 8$ ) are open. (Bottom) Gap voltage waveform with the feedback loops closed for all harmonics.

harmonic $(h=3)$ of the driven harmonic has a large amplitude of $0.8 \mathrm{kV}$, and the second harmonic $(h=2)$ has an amplitude of a few percent of the driven harmonic. The amplitudes of the other harmonics are negligibly small. One can see that the distortion shown in the top plot in Fig. 24 is mainly due to the third harmonic.

By closing the feedback loops for the higher harmonics with the set point of $(I, Q)=(0 \mathrm{kV}, 0 \mathrm{kV})$, the higher harmonics are suppressed as plotted in the bottom plot in Fig. 25. The maximum amplitude of the second harmonic $(h=2)$ is $0.1 \mathrm{kV}$, and the other harmonics are suppressed below $0.05 \mathrm{kV}$. The voltage waveform is very close to the pure sinusoidal as shown in the bottom plot in Fig. 24.
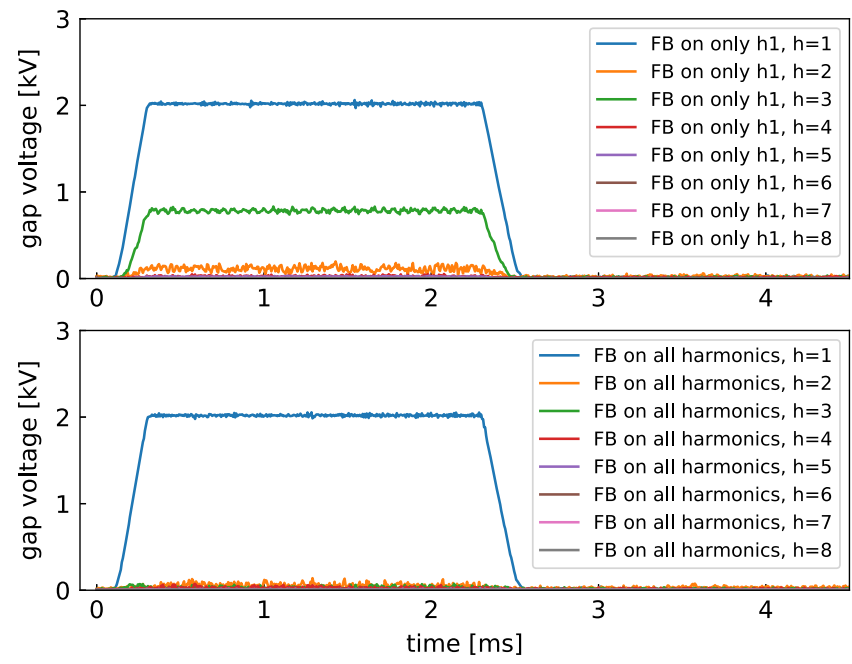

FIG. 25. Harmonic components of the gap voltage (top) when only the feedback for $(h=1)$ is closed and the other harmonics ( $h=2 \ldots 8$ ) are open. (Bottom) With the feedback loops closed for all harmonics. 

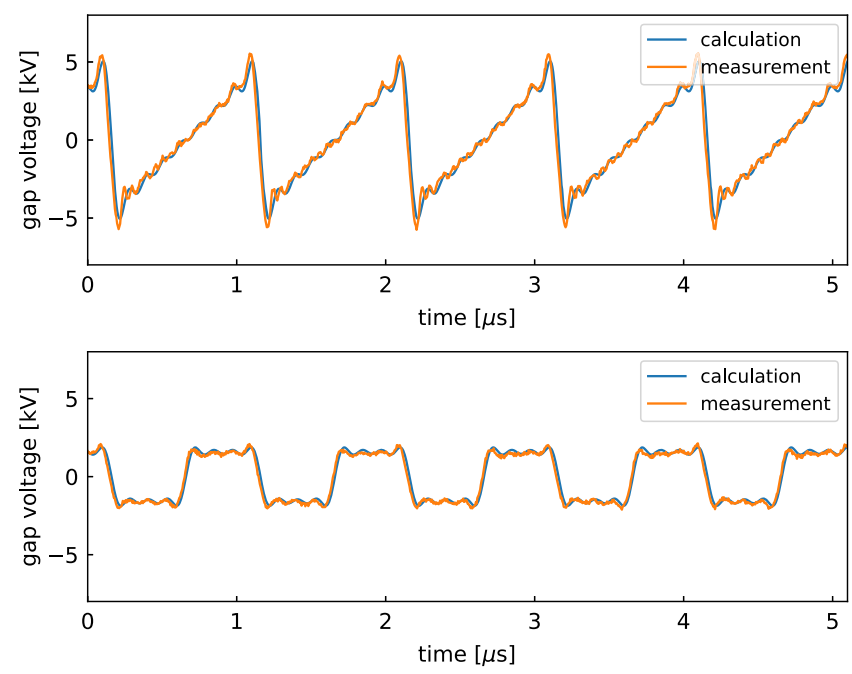

FIG. 26. Comparison of the calculated and measured gap voltage waveforms of (top) a sawtooth wave and (bottom) a square wave.

By this test, the performance of the voltage control for reduction of distortion is confirmed.

\section{B. Multiharmonic rf voltage generation}

To demonstrate the performance of the multiharmonic vector rf voltage control, sawtooth and square waves are generated. In the demonstration, the frequency is set to $1 \mathrm{MHz}$. The $I / Q$ amplitudes of $(h=1)$ are set to $(0 \mathrm{kV}$, $3 \mathrm{kV})$ and $(0 \mathrm{kV}, 1 \mathrm{kV})$ for the sawtooth and square waves, respectively. The amplitudes of the higher harmonics are set according to the Fourier coefficients. The generated waveforms are compared with the calculated waveforms using the Fourier coefficients in Fig. 26. The maximum deviation of the harmonic amplitudes is on the order of $0.02 \mathrm{kV}$. The waveforms agree very well for both the sawtooth and square waves.

The performance of the multiharmonic voltage generation is promising. The longitudinal painting [41] during the injection period is currently performed by using two harmonics $(h=2,4)$. We consider that the higher harmonics $(h=6,8)$ may be used in addition for improvement of the longitudinal painting.

\section{BEAM TEST RESULTS}

\section{A. Cancellation of the wake voltages without driving rf}

The test for cancellation of the wake voltage without driving $\mathrm{rf}$ was conducted to demonstrate the performance of the multiharmonic vector rf voltage control for suppression of the wave voltages, although the situation that one of the cavities is turned off is not a normal operating condition.

The setup of the beam test is illustrated in Fig. 27. Cavity 5 is used for the beam test. The driving signal and the feedforward compensation signal from the existing LLRF

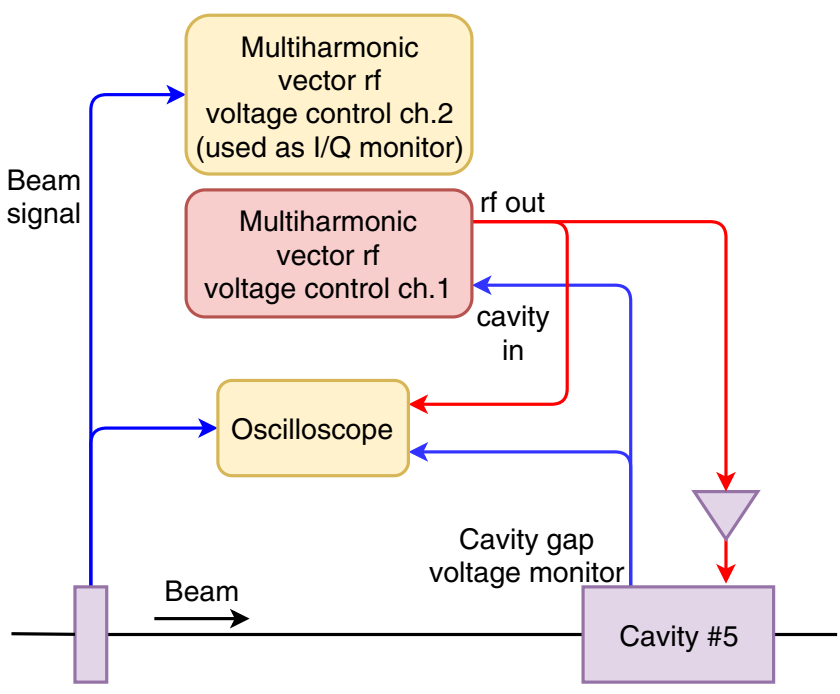

wall current monitor

FIG. 27. Beam test setup.

control system are disconnected for the tests. Thus, the gap voltage of cavity 5 is composed by the wake voltage induced by the beam and the compensation signal generated by the voltage control. The output signal of the module, the beam signal from the wall current monitor, and the cavity gap voltage monitor signal are recorded by an oscilloscope from injection to extraction. All the other cavities are connected to the existing LLRF control system as the normal operation. The $I / Q$ amplitudes for eight harmonics of the cavity gap voltages and the beam signal are recorded by the internal $I / Q$ monitor in the system.

In this situation, the beam intensity is limited by the reduced total accelerating voltage and the rf bucket distortion due to the wake voltage in cavity 5 . The maximum beam intensities without significant intensity loss are $4.2 \times 10^{13}$ and $2.1 \times 10^{13} \mathrm{ppp}$ for the cases of the acceleration of two bunches and a single bunch, respectively. The harmonic components of the beam in these cases are plotted in Fig. 28.

The beam is injected at $-250 \mu$ s before the bottom of the sinusoidal magnetic ramping, which is called $B_{\min }$, for $500 \mu \mathrm{s}$. In the case of the acceleration of two bunches, the beam contains only even harmonics $(h=2,4,6,8)$. All eight harmonics are seen in the case of the single-bunch acceleration.

The harmonic components of the cavity gap voltage from injection to extraction without and with feedback are plotted in Fig. 29. The voltage waveforms just before extraction, 19.9 ms, are plotted in Fig. 30.

Significant harmonic amplitudes of the wake voltage are observed without feedback. The fundamental accelerating harmonic $(h=2)$ and the second harmonic $(h=4)$ of the wake voltage reach almost 7 and $2 \mathrm{kV}$ just before the extraction, respectively. Because of the cavity frequency response, the higher harmonics $(h=6,8)$ are relatively small, 0.7 and $0.2 \mathrm{kV}$, respectively. 

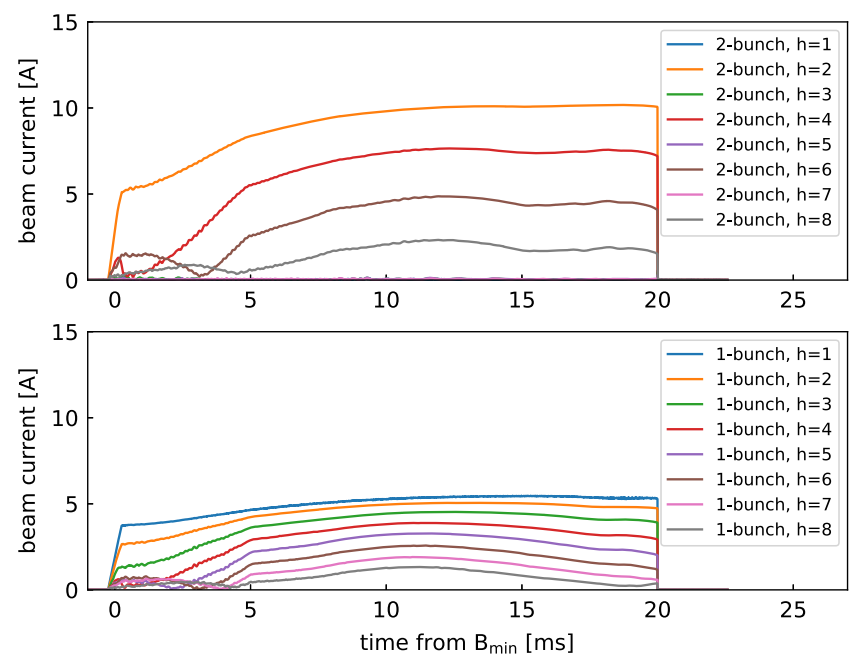

FIG. 28. Harmonic components of the beam current in the cases of (top) acceleration of two bunches at $4.2 \times 10^{13} \mathrm{ppp}$ and (bottom) acceleration of a single bunch at $2.1 \times 10^{13} \mathrm{ppp}$.

The gap voltage waveform is the superposition of the harmonics. As shown in Fig. 30, the wake voltage has a distorted waveform, which contains several harmonics. The voltage swing has a large amplitude from -10 to $8 \mathrm{kV}$.

By closing the feedback with the set point of $(I, Q)=$ $(0 \mathrm{kV}, 0 \mathrm{kV})$ for all eight harmonics, the harmonics components of the gap voltage are suppressed to less than $0.15 \mathrm{kV}$ throughout the accelerating period as shown in the middle and bottom plots in Fig. 29. A voltage jump
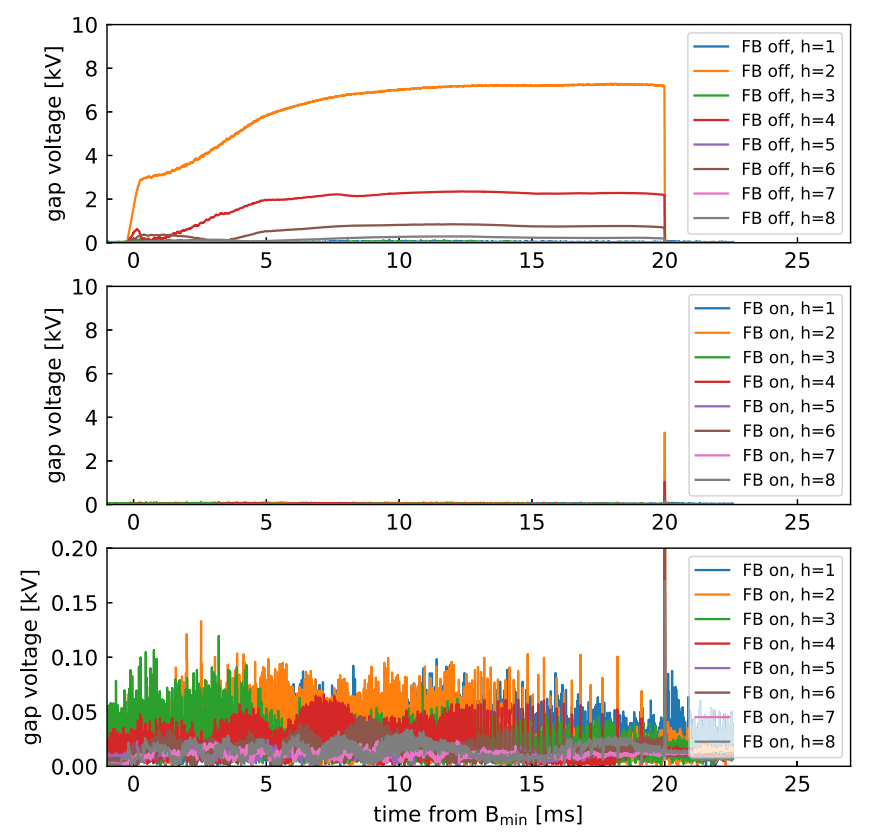

FIG. 29. Harmonic components of the gap voltage during acceleration of two bunches (top) without feedback and (middle) with feedback and (bottom) a vertically magnified view of the middle plot.

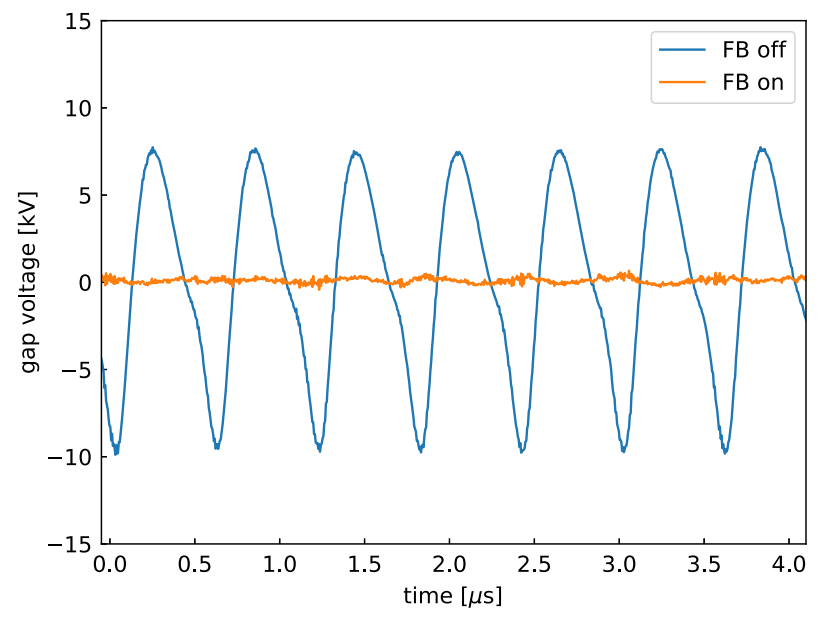

FIG. 30. Comparison of the gap voltage waveforms in the case of acceleration of two bunches without and with feedback just before extraction at $19.9 \mathrm{~ms}$.

at $20 \mathrm{~ms}$ due to the delay of the feedback loop is observed. The beam is extracted by the kicker magnet in one turn, and the amplitude of the wake voltage suddenly changes to zero. Because of the delay of the feedback loop, the output amplitude of the voltage control decays as the step response of the loop. It takes several tens of microseconds to reach down to zero $\mathrm{kV}$. The countermeasure for this unwanted voltage jump is discussed in the following subsection (Sec. VIC). The amplitude of voltage waveform just before extraction is less than $0.3 \mathrm{kV}$, as shown in Fig. 30 .

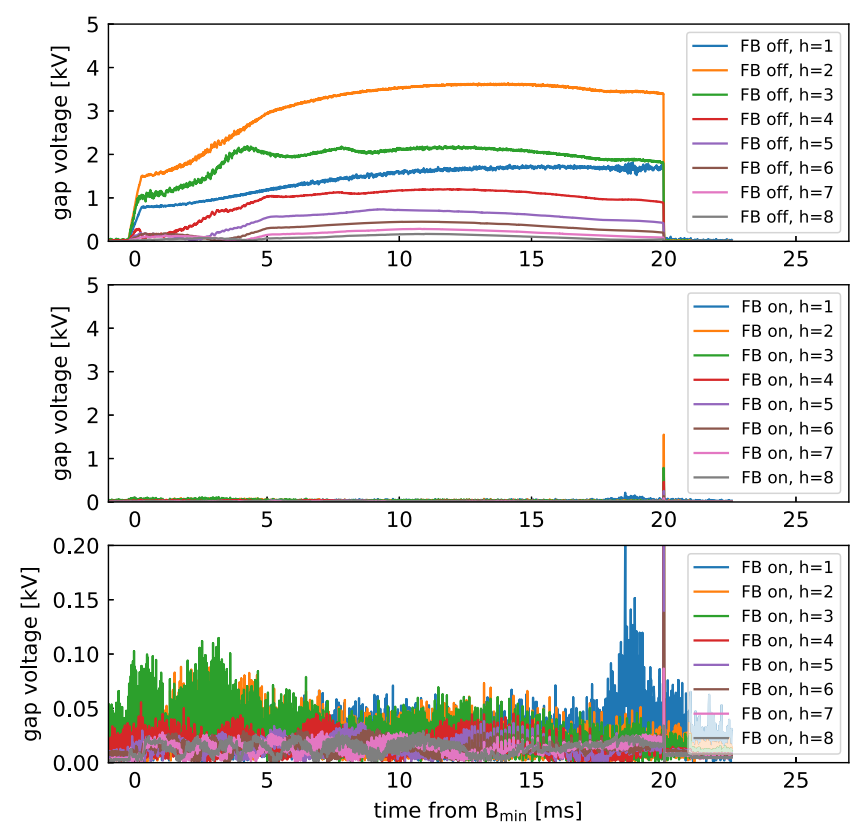

FIG. 31. Harmonic components of the gap voltage during acceleration of a single bunch (top) without feedback and (middle) with feedback and (bottom) a vertically magnified view of the middle plot. 


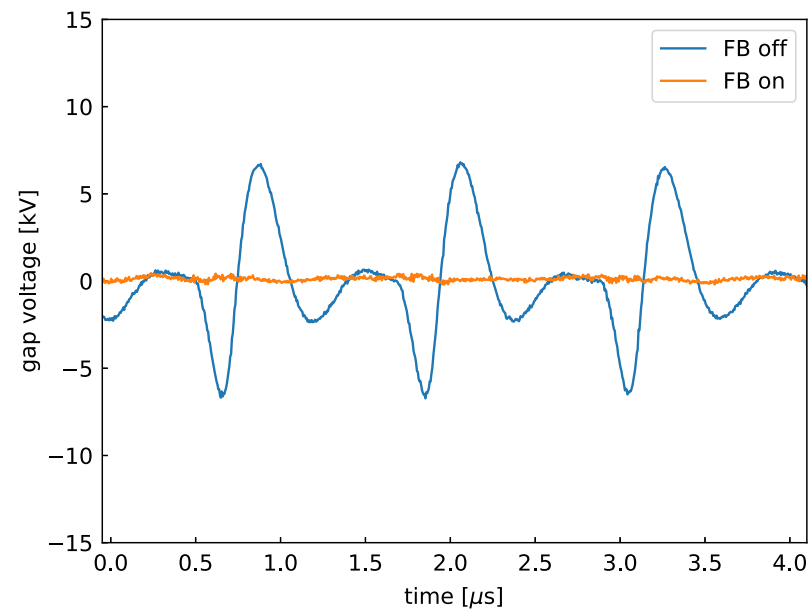

FIG. 32. Comparison of the gap voltage waveforms in the case of acceleration of a single bunch without and with feedback just before extraction at $19.9 \mathrm{~ms}$.

In the case of the single-bunch acceleration, the wake voltage contains the odd harmonics $(h=1,3,5,7)$ as well as the even harmonics, as shown in the top plot in Fig. 31. The amplitudes of $(h=1,3)$ are relatively high among the odd harmonics. The amplitudes of the significant harmonics
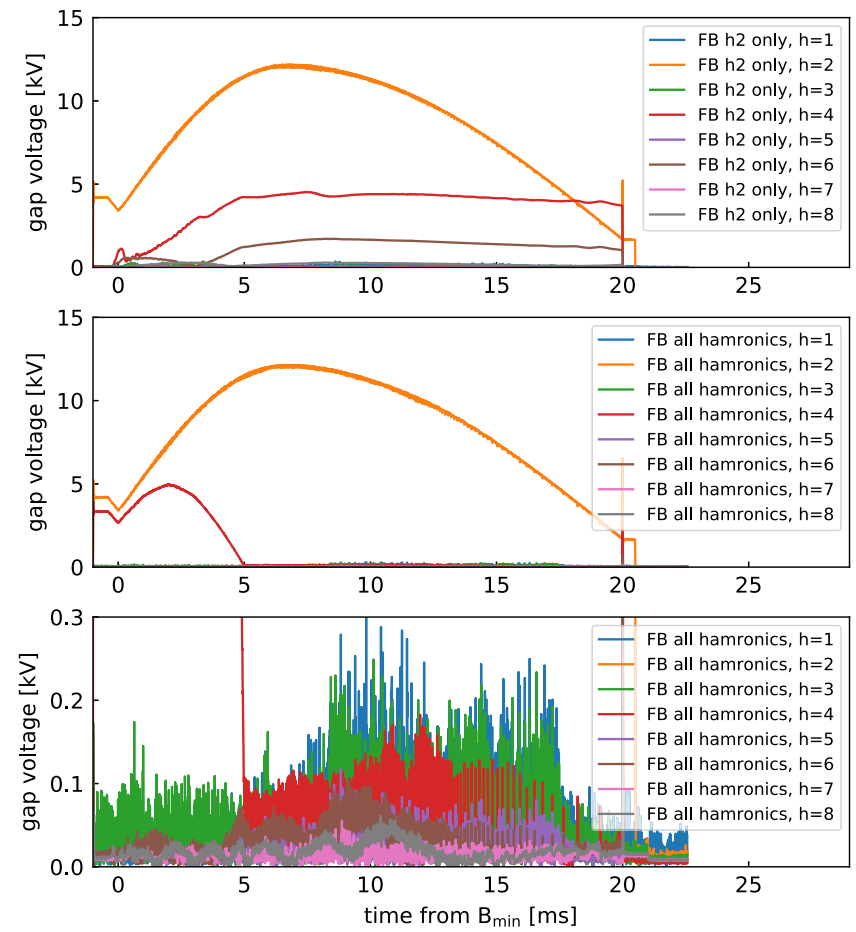

FIG. 33. Amplitudes of the harmonic components with an acceleration of a $1 \mathrm{MW}$ equivalent beam from injection to extraction in the cases where (top) the feedback for the fundamental acceleration harmonic $(h=2)$ is closed and the output of the others is turned off and (middle) the feedbacks for all harmonics are closed and (bottom) a vertically magnified view of the middle plot. $(h=1,2,3)$ are $3.3,2$, and $1.8 \mathrm{kV}$, respectively. Since it consists of more harmonics than the case of the acceleration of two bunches, the voltage waveform as plotted in Fig. 32 is more distorted than the case of the acceleration of two bunches. The amplitude of the voltage waveform exceeds $6 \mathrm{kV}$. Because of the components of odd harmonics, the repetition period of the voltage waveform is the beam revolution period.

With feedback, the wake voltage is nicely canceled. All of the harmonics except $(h=1)$ of the gap voltage are less than $0.15 \mathrm{kV}$ as shown in the middle and bottom plots in Fig. 31. The amplitude of $(h=1)$ reaches $0.2 \mathrm{kV}$ around $19 \mathrm{~ms}$, while it is less than $0.1 \mathrm{kV}$ other than that. The voltage waveform just before extraction in Fig. 32 is quiet with a remaining amplitude of less than $0.3 \mathrm{kV}$, as well as in the case of two bunches plotted in Fig. 30.

Thus, a significant reduction of the wake voltages was demonstrated for both cases of the acceleration of the single bunch and two bunches, where the operating conditions of the vacuum tube are expected to be different.

\section{B. Beam loading compensation with driving rf voltages}

Beam loading compensation with driving accelerating rf voltage was tested as a realistic situation. In this case, the beam is accelerated by the voltages of the cavities controlled by the existing LLRF control system and cavity 5
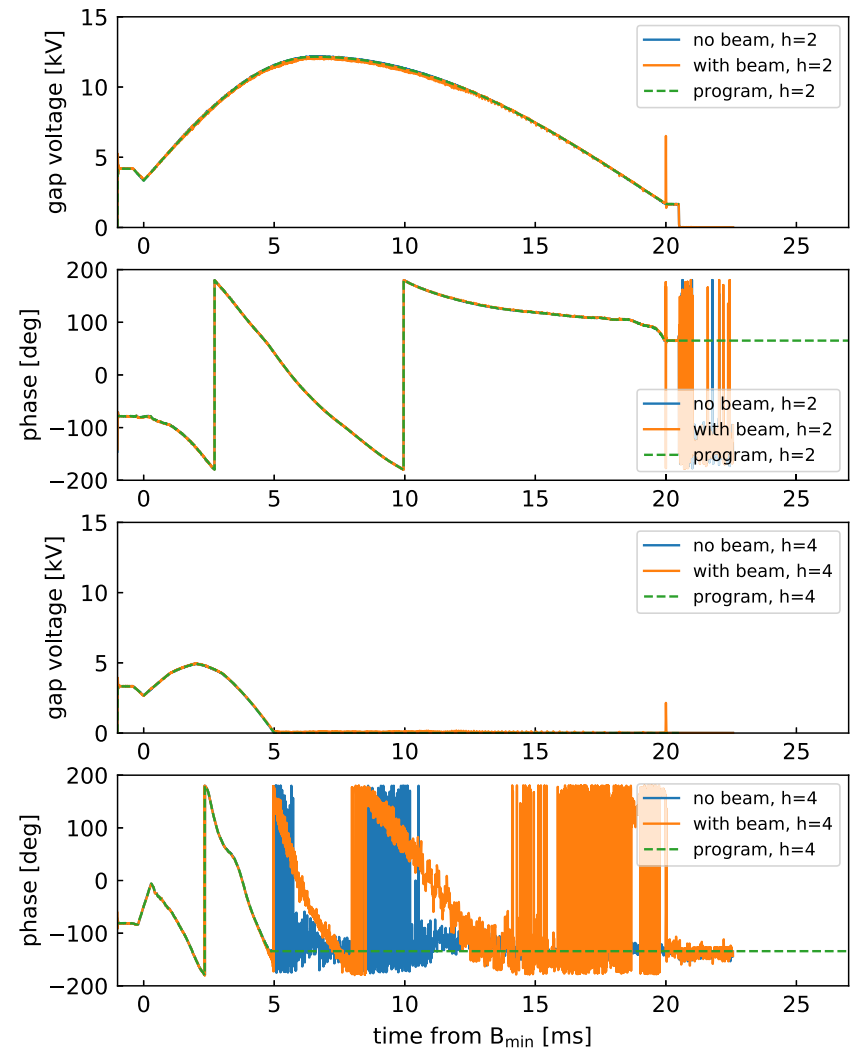

FIG. 34. Comparison of the amplitudes and phases of the driven harmonics $(h=2,4)$ to the programs without and with a beam. 


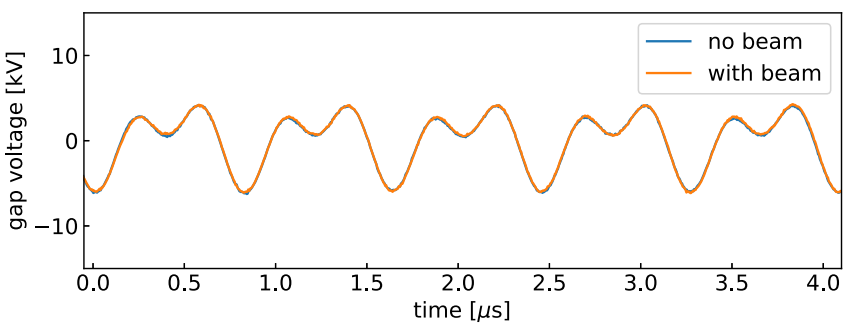

(a) $0 \mathrm{~ms}$.

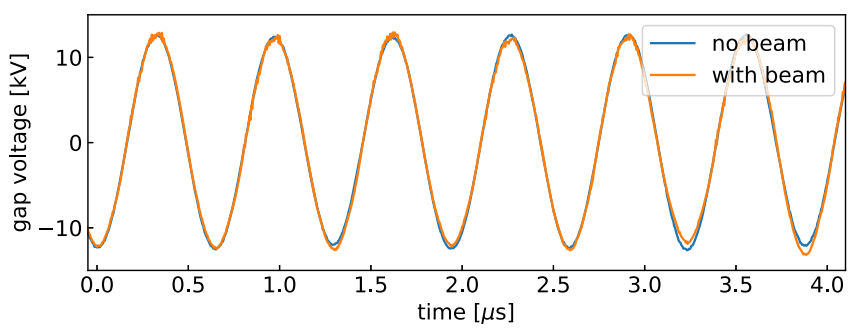

(c) $8 \mathrm{~ms}$.

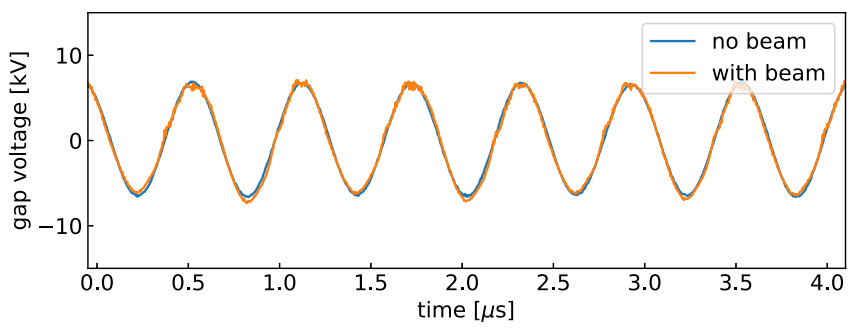

(e) $16 \mathrm{~ms}$.

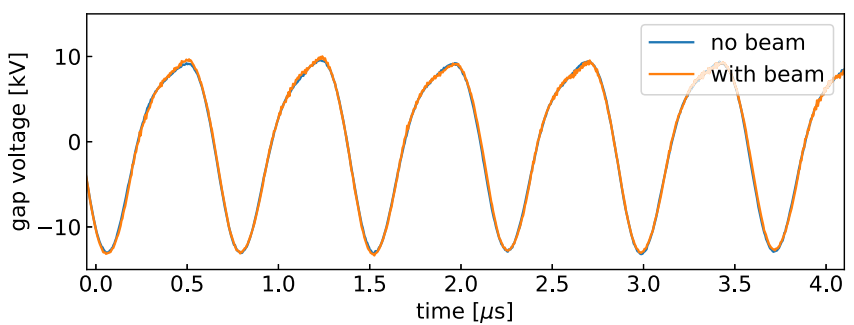

(b) $4 \mathrm{~ms}$.

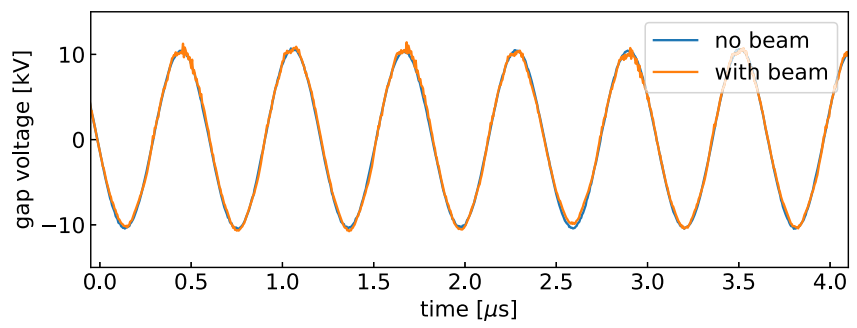

(d) $12 \mathrm{~ms}$.

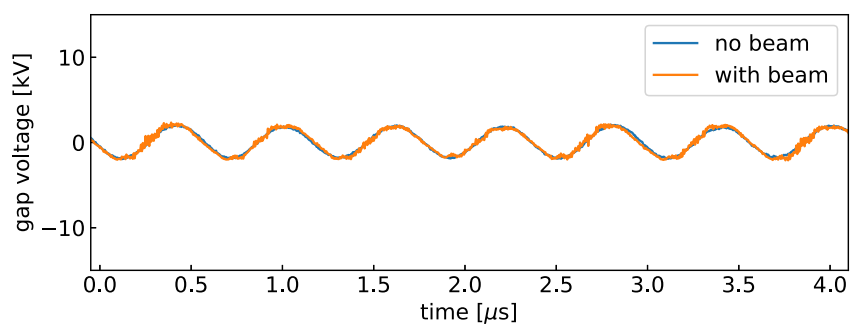

(f) $19.9 \mathrm{~ms}$.

FIG. 35. Comparison of the gap voltage waveforms without and with a beam during acceleration.

with the new system. The $I / Q$ voltage patterns for the driving $\mathrm{rf}(h=2,4)$ are set by using the measured phases of the voltage controlled by the existing system, so that the total accelerating voltage is similar to the acceleration with the existing system.

High-intensity beams of up to $8.3 \times 10^{13} \mathrm{ppp}$, which is the design intensity and corresponds to a beam power of $1 \mathrm{MW}$ at a repetition rate of $25 \mathrm{~Hz}$, are used for the test.

The harmonic components of the gap voltage from injection to extraction for the acceleration of a $1 \mathrm{MW}$ equivalent beam are plotted in Fig. 33. The top plot shows the harmonic components in the case that the feedback for the fundamental acceleration harmonic $(h=2)$ is closed and the others are open. The second harmonic $(h=4)$ voltage is not driven. Because the beam contains only even harmonics when two bunches are accelerated, the even harmonics are observed in the gap voltage. The fundamental accelerating harmonic $(h=2)$ is regulated according to the voltage program. The amplitudes of $(h=4,6)$ are significant, 4.6 and $1.7 \mathrm{kV}$ maximum, respectively. These harmonic components $(h=4,6)$ mainly consist of the wake voltage but also contain the distortion of the vacuum tube output current when the compensation signal of the fundamental harmonic $(h=2)$ has a large amplitude. The $(h=8)$ component is observed, but the amplitude is very small.

The harmonic components of the gap voltage when the feedbacks for all harmonics are closed are plotted in the middle in Fig. 33. The vertically magnified view is shown in the bottom plot. The amplitude of the fundamental accelerating harmonic $(h=2)$ is the same as in the top plot. In contrast to the top plot, here the second harmonic $(h=4)$ has a voltage program from the beginning to $5 \mathrm{~ms}$. After $5 \mathrm{~ms}$, the voltage program is zero and the amplitude of the $(h=4)$ harmonic is suppressed to less than $0.2 \mathrm{kV}$. The other harmonics that are not used for acceleration and have voltage programs of zero are well kept less than $0.3 \mathrm{kV}$ throughout the acceleration.

Thus, the suppression of the wake voltage for the harmonics that are not used for acceleration was confirmed as well as in the case that the cavity is not driven described in the previous subsection. One should note that the output currents of the vacuum tube are very different between these situations and the tube gains are expected to be different. The feedback works nicely for both cases.

The amplitudes and phases of the driven harmonics $(h=2,4)$ without and with a beam are compared to the programs in Fig. 34. The amplitudes of the fundamental 
accelerating harmonic $(h=2)$ without and with a beam are very close to the program. A maximum deviation of $0.1 \mathrm{kV}$, which corresponds to $0.8 \%$ of the program voltage, is observed near the voltage peak around $7 \mathrm{~ms}$ with beam. The unwanted voltage jump just after the extraction is also seen. The phase of $(h=2)$ is well regulated within less than $1^{\circ}$ from the program without and with a beam. The amplitudes of the second harmonic $(h=4)$ without and with a beam are also very close to the program. It follows the voltage program nicely within $0.03 \mathrm{kV}$ until $5 \mathrm{~ms}$ and is kept less than $0.2 \mathrm{kV}$ after $5 \mathrm{~ms}$. The deviations of the phase of $(h=4)$ without and with a beam from the program are less than $1^{\circ}$ until $5 \mathrm{~ms}$. After $5 \mathrm{~ms}$, the comparison does not make sense, because the programed amplitude is zero. Thus, the voltages of the driving harmonics are generated as programed under the heavy beam loading of the $1 \mathrm{MW}$ equivalent beam.

The voltage waveforms without and with a beam are compared at several points during the acceleration. The corresponding plots are generated every $4 \mathrm{~ms}$ and are shown in Fig. 35. In all plots, the waveforms without and with a beam nicely agree. The second harmonic $(h=4)$ has a nonzero voltage program until $5 \mathrm{~ms}$ for the longitudinal painting, and the waveforms in the first two plots (a) and (b) contain the second harmonic voltages. After $5 \mathrm{~ms}$, the expected waveform is sinusoidal. No waveform delays due to the beam loading are observed. The bunch width becomes narrower with acceleration from about 450 to $150 \mathrm{~ns}$, and the peak current becomes higher. Higher-frequency components are observed in the voltage waveform after $12 \mathrm{~ms}$.

The measurements described here prove that the heavy beam loading of a $1 \mathrm{MW}$ equivalent beam in the cavity including the higher harmonics is almost perfectly compensated by the multiharmonic vector rf voltage control. The large amplitude compensation signal is supposed to generate the gap voltage distortion, and this is also compensated by the voltage control.

The beam intensity for the single-bunch acceleration is limited to $3.1 \times 10^{13} \mathrm{ppp}$, which is $24 / 32$ of the full intensity, due to the output saturation of the voltage control. When accelerating the very-high-intensity beams with multiharmonic beam loading compensation, the output of the solid-state amplifier is close to the maximum rating. As shown in Fig. 5, the solid-state amplifier has a nonlinearity and saturation behavior near the maximum rating. We suspect that the solid-state amplifier introduces additional distortion near the maximum rating, and its compensation requires more amplitude of the voltage control output. This requires more output of the solid-state amplifier; therefore, more distortion to be compensated occurs at the accelerating gap. Finally, the output of the voltage control saturates. The consolidation of the amplifier chain, especially the solid-state amplifier, is demanded to alleviate the unwanted output saturation. We are considering and investigating a possible consolidation.
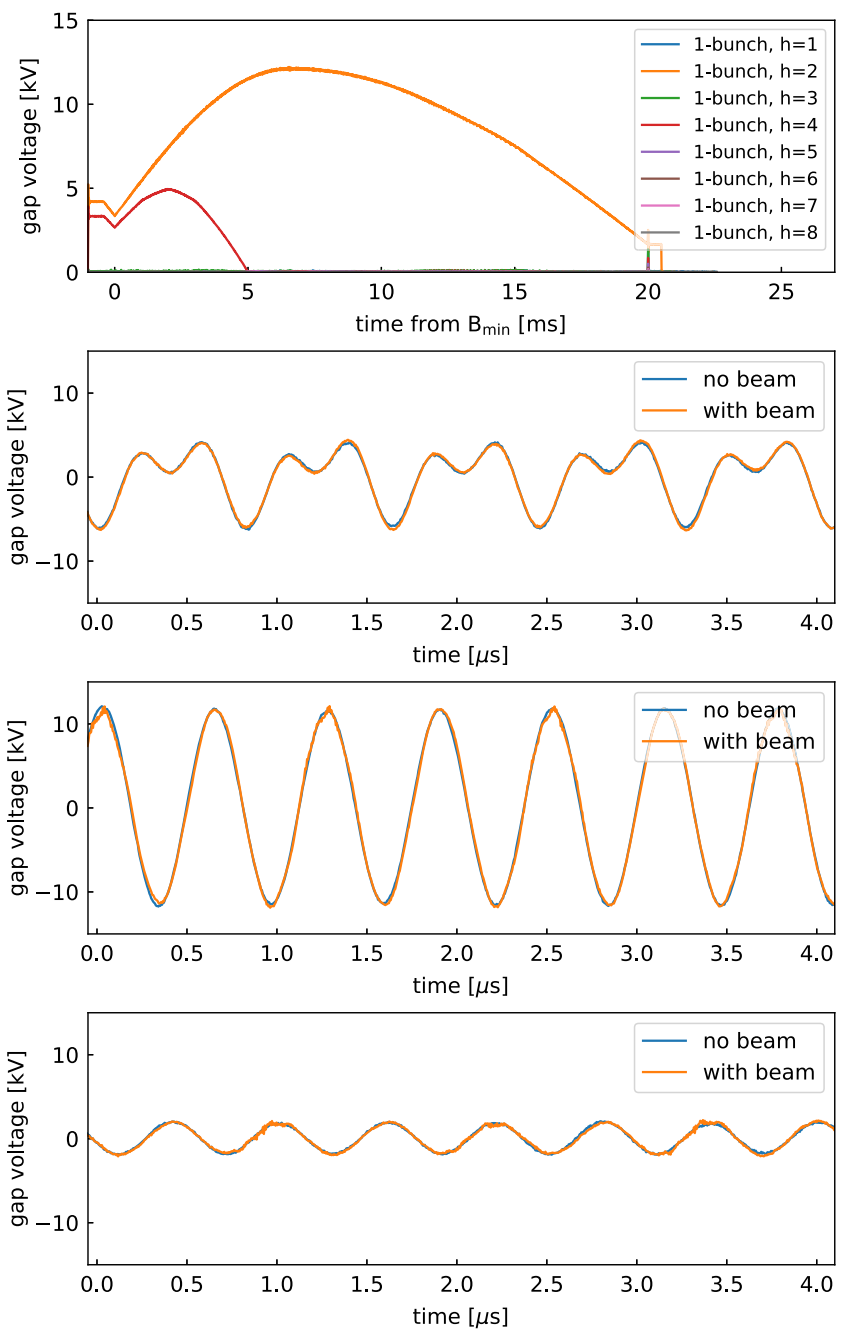

FIG. 36. (Top) Amplitudes of the harmonic components with acceleration of a single bunch at $3.1 \times 10^{13} \mathrm{ppp}$ from injection to extraction and (second top to bottom) comparison of the gap voltage waveforms without and with a beam at 0,10 , and $19.9 \mathrm{~ms}$.

Below the intensity limit, the beam loading of the singlebunch beam is nicely compensated as well as the case of the acceleration of two bunches. The top plot in Fig. 36 shows the amplitudes of the harmonic components with acceleration of a single bunch at $3.1 \times 10^{13} \mathrm{ppp}$. The driving harmonics $(h=2,4)$ are generated as programed, and the other harmonics are suppressed below $0.1 \mathrm{kV}$. The waveforms without and with a beam are compared at 0,10 , and $19.9 \mathrm{~ms}$ in the figure. They almost completely agree.

\section{Mitigation of the unwanted voltage jump by applying the gain pattern}

As described in the previous subsection, the gap voltage jumps up just after the beam extraction. As shown in Fig. 37, the gap voltage exceeds $17 \mathrm{kV}$ when a $1 \mathrm{MW}$ equivalent beam is extracted. It is higher than the maximum acceleration voltage of $12.2 \mathrm{kV}$. Even though the duration 


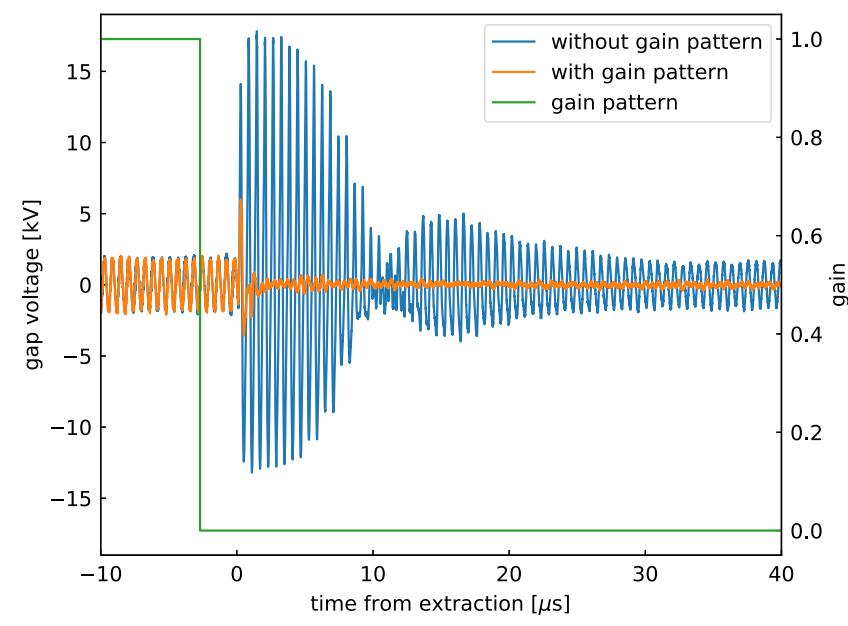

FIG. 37. Voltage waveforms near the extraction of a $1 \mathrm{MW}$ equivalent beam without and with the gain pattern. The applied gain pattern is also plotted.

of the high voltage is short, less than $5 \mu$ s, the voltage jump may reduce the lifetime of the gap capacitors.

The voltage jump occurs because of the delay of the feedback. The steady state phasor diagram [5] is illustrated in Fig. 38(a). The total current $I_{T}$ into the cavity is the vector sum of the generator current $I_{g}$ and the beam current $I_{b}$, which have the phase angles of $\phi_{b}$ and $\phi_{L}$, respectively. $\phi_{L}$ is the beam loading angle. $I_{0}$ is the resistive current into the cavity, where $I_{0}=I_{T} \cos \phi_{z}$. The voltage is proportional to $I_{T}$ and $I_{0}$, where $\phi_{z}$ is the detuning angle of the cavity. $I_{g}$ is controlled by the feedback so that the gap voltage is as programed. With high-intensity beams, namely, under heavy beam loading with $Y=6.9,\left|I_{g}\right|$ is much larger than $\left|I_{T}\right|$. We put $I_{g}=I_{g 0}$ just before extraction.

When the beam is extracted by the kicker magnets in a single turn, the beam current becomes suddenly zero. The phasor diagram just after extraction is shown in Fig. 38(b). The new total current into the cavity $I_{T}^{\prime}$ consists of only the generator current. Because of the system delay, the new generator current is still kept as $I_{g 0}$ before extraction. Therefore, the new total current $I_{T}^{\prime}=I_{g 0}$, which has a larger amplitude $\left|I_{T}^{\prime}\right|>\left|I_{T}\right|$ and a different phase angle, is fed to the cavity. The new resistive current $I_{0}^{\prime}=I_{T}^{\prime} \cos \phi_{z}$ is also larger than $I_{0}$. Since the RCS cavity has a very low $Q$ value of 2 , the voltage reacts quickly within a few cycles. That is the reason why the gap voltage jumps up just after extraction.

While the delay of the feedback is inevitable, the voltage jump can be mitigated by applying the gain pattern, which is included in Fig. 6. Since the timing of the MLF beam extraction is fixed [42], one can turn off the generator current when the beam is extracted by turning off the rf output by the gain pattern just before extraction of the beam. The timing must be adjusted carefully. If the change is too early, the suppression of the wake voltage becomes worse before extraction, and the beam may be affected by

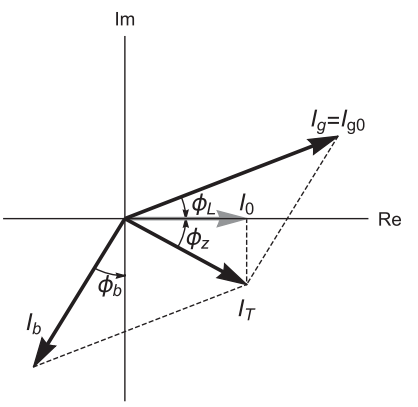

(a)

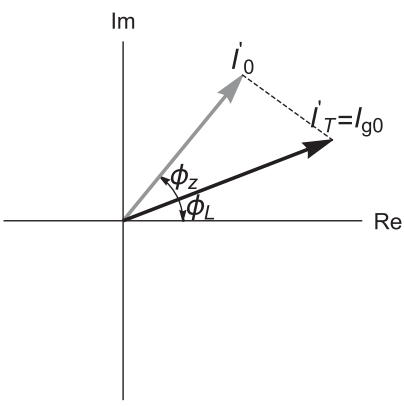

(b)
FIG. 38. (a) Steady state phasor diagram under beam loading and (b) phasor diagram just after the beam extraction.

the wake voltage. If the change is late, the voltage jump remains. The sampling frequency of the gain pattern is $1 \mathrm{MHz}$, which gives a timing resolution of $1 \mu \mathrm{s}$.

The timing is adjusted by looking at the voltage waveform. The optimum timing is from $19.995 \mathrm{~ms}$ from the $B_{\min }$, which is $3.7 \mu$ s before extraction. The applied gain pattern and resultant voltage waveform are plotted in Fig. 37. The unwanted voltage jump is well mitigated.

This method cannot be applied for the MR beam extraction, where the extraction timing varies within on the order of $10 \mu$ s for selection of the MR bucket. We are considering a countermeasure against the voltage jump for the MR beam extraction.

\section{CONCLUSION}

We developed a novel multiharmonic vector rf voltage control for the wideband MA cavity driven by a vacuum tube amplifier as a part of the next-generation LLRF control system for the J-PARC RCS. It controls the complex amplitudes of eight harmonics to cover the wide frequency response of the cavity, where multiharmonic beam loading must be compensated for high-intensity beam acceleration.

Originally, we employed the rf feedforward method for beam loading compensation, and the multiharmonic feedforward works fairy well with high-intensity beams up to the design beam power of $1 \mathrm{MW}$, while the degradation of its compensation performance has been observed with more than $600 \mathrm{~kW}$ equivalent beams. Circuit simulations are performed to address the issues of the rf feedforward method. The gain variation and output current distortion of the vacuum tube introduce difficulties of the feedforward.

The multiharmonic vector rf voltage control is composed by the $I / Q$ feedback blocks with features for the frequency sweeps during the proton beam acceleration. The phase offset LUT compensates the phase shift due to the long cable to keep the condition for closing the feedback, and the gain LUT compensates the amplitude response of the cavity so that similar feedback gains are obtained for all harmonics. The commissioning methodology of the LUTs is established. 
The performance of the feedback strongly depends on the configuration of the low-pass filter in the $I / Q$ demodulator. The hybrid filter, where the tracking CIC filter and the leaky integrator are cascaded, is finally selected with a detailed analysis. By both simulations and the measurements, the advantage of the hybrid filter on the performance concerning the feedback over the five-stage CIC filter is confirmed.

Driving of the wideband cavity with the vacuum tube amplifier causes voltage distortion, since the output current of the tube contains higher harmonics. It becomes significant when the output current of the tube is high, in other words, under the heavy beam loading that requires large amplitude compensation signals. The multiharmonic vector rf voltage control suppresses the voltage distortion nicely. Recently, the wideband MA cavities were used in the CERN accelerator complex [22] and a similar $I / Q$ feedback loop was employed, where they call it the cavity servo loop [43]. The major difference between us and CERN is that they employ the low-distortion solid-state amplifier as the final stage amplifier. In our case, the vacuum tube amplifier is necessary because of the very high required voltage and power. The multiharmonic vector rf voltage control works with the vacuum tube amplifier.

The successful demonstration of the multiharmonic of voltage generation gives us the opportunity to consider the improvement of the longitudinal painting during the injection period using more than two harmonics, which is the situation at present.

Beam tests using very-high-intensity beams up to the design intensity of $8.3 \times 10^{13} \mathrm{ppp}$ that corresponds to $1 \mathrm{MW}$ at the repetition rate of $25 \mathrm{~Hz}$ were successfully carried out. In the tests for cancellation of the wake voltages without driving rf, the wake voltages of significant amplitudes induced by the high-intensity beam are well suppressed for both cases of the acceleration of two bunches and a single bunch, where the operating conditions of the vacuum tube are different. With driving rf voltages, the beam loading of the $1 \mathrm{MW}$ equivalent beam is compensated. The amplitude and phase of the driving harmonics $(h=2,4)$ are controlled as programed. The other harmonics due to the wake voltage induced by the beam and also due to the distortion of tube output current are significantly suppressed. We emphasize that the clean rf voltage in the cavity as programed is very helpful for transverse beam dynamics studies, since it is difficult to include the beam loading effects or the voltage distortion due to the tube in the transverse space charge tracking simulation codes. The clean rf voltage is helpful to compare the real beams with simulations.

We note here that the compensation of the odd harmonics is important to accelerate two bunches at very high intensity, because they may cause unwanted beam oscillations in some conditions [12].

The intensity for the single-bunch acceleration is limited to $3.1 \times 10^{13}$ ppp because of the output saturation of the voltage control. The consolidation of the amplifier chain is demanded. As described in Sec. II, the solid-state amplifier in the chain is a possible major source of the saturation. A consolidation plan is under consideration.

The inevitable delay of the feedback causes the unwanted voltage jump just after the extraction of high-intensity beams. It is mitigated by applying a suitable gain pattern in the feedback block.

The performance of the multiharmonic vector voltage control is promising. It works well for various beam conditions, where the operating conditions of the vacuum tube are expected to be also various. The existing LLRF control system for the RCS will be replaced with the nextgeneration system during the summer maintenance period in 2019. Having multiharmonic vector rf voltage control for all 12 cavities, more stable acceleration of the highintensity beams is expected.

\section{ACKNOWLEDGMENTS}

We thank H. Damerau, M. Paoluzzi, J. Molendijk, and M. E. Angoletta for fruitful discussions and comments. The nice and perfect job for implementation of the nextgeneration LLRF control system by Mitsubishi Electric Tokki Systems Corporation is appreciated. We thank A. Schnase for his useful and detailed suggestions. We also thank the beam commissioning group of the RCS headed by $\mathrm{H}$. Hotchi for providing the beam time for the tests of the system. We are grateful to the J-PARC writing support group, which continuously encouraged us to write up this article. Also, we thank all the members of the J-PARC. We acknowledge the late Prof. Yong Ho Chin for his activity in accelerator physics and technology which was inspirational.

[1] JHF Project Office, JHF accelerator design study report, KEK-Report No. 97-16, 1997.

[2] High-Intensity Proton Accelerator Project Team, Accelerator technical design report for J-PARC, JAERI-TECH Report No. 2003-044, 2003.

[3] H. Hotchi, H. Harada, N. Hayashi, S. Kato, M. Kinsho, K. Okabe, P. K. Saha, Y. Shobuda, F. Tamura, N. Tani, Y. Watanabe, K. Yamamoto, M. Yamamoto, and $M$. Yoshimoto, Achievement of a low-loss 1-MW beam operation in the $3-\mathrm{GeV}$ rapid cycling synchrotron of the Japan Proton Accelerator Research Complex, Phys. Rev. Accel. Beams 20, 060402 (2017).

[4] P. B. Wilson, Beam loading in high-energy storage rings, in Proceedings of the 9th International Conference on the High-Energy Accelerators (HEACC 1974), Stanford, California, 1974 (SLAC, Stanford, CA, USA, 1974), pp. 57-62.

[5] F. Pedersen, Beam loading effects in the CERN PS booster, IEEE Trans. Nucl. Sci. 22, 1906 (1975).

[6] D. Boussard, Control of cavities with high beam loading, in Proceedings of the 11th Particle Accelerator 
Conference, PAC-1985, Vancouver, BC, Canada, 1985 (IEEE, New York, 1985), pp. 1852-1856.

[7] F. Pedersen and F. Sacherer, Theory and performance of the longitudinal active damping system for the CERN ps booster, IEEE Trans. Nucl. Sci. 24, 1396 (1977).

[8] S. Stahl and S. A. Bogacz, Coupled-bunch instability in a circular accelerator and possible cures: Longitudinalphase-space simulation, Phys. Rev. D 37, 1300 (1988).

[9] H. Damerau et al., Longitudinal coupled-bunch instabilities in the CERN PS, in Proceedings of the 22nd Particle Accelerator Conference, PAC-2007, Albuquerque, NM (IEEE, New York, 2007), pp. 4180-4182.

[10] H. Damerau et al., Longitudinal coupled-bunch oscillation studies in the CERN PS, in Proceedings of the 4th International Particle Accelerator Conference, IPAC2013, Shanghai, China, 2013 (JACoW, Shanghai, China, 2013), pp. 1808-1810.

[11] Y. Shobuda (private communication).

[12] M. Yamamoto, F. Tamura, M. Nomura, T. Shimada, C. Ohmori, K. Hasegawa, K. Hara, M. Yoshii, and Y. Sugiyama, Observation of simultaneous oscillations of bunch shape and position caused by odd-harmonic beam loading in the Japan Proton Accelerator Research Complex Rapid Cycling Synchrotron, Prog. Theor. Exp. Phys. (2017), 113G01.

[13] M. Yamamoto et al., Beam loading effects in JHF synchrotron, in Proceedings of the Asian Particle Accelerator Conference (APAC 98) (KEK, Tsukuba, Japan, 1998), p. 398.

[14] K. Saito et al., Higher harmonics beam loading compensation for a broad band MA-loaded rf cavity, in Proceedings of the Asian Particle Accelerator Conference (APAC 98) (KEK, Tsukuba, Japan, 1998), p. 399.

[15] M. Yamamoto, Longitudinal particle tracking code for a high intensity proton synchrotron, in Proceedings of the ICFA Advanced Beam Dynamics Workshop on HighIntensity and High-Brightness Hadron Beams (HB'16), Malmo, Sweden, 2016 (JACoW, Geneva, 2016), Vol. 57, pp. 110-114.

[16] Nanocrystalline soft magnetic material, FINEMET, http:// www.hitachi-metals.co.jp/e/products/elec/tel/p02_21.html.

[17] C. Ohmori et al., High field-gradient cavities loaded with magneticalloys for synchrotrons, in Proceedings of the 18th Particle Accelerator Conference, New York, 1999 (IEEE, New York, 1999), pp. 413-417.

[18] H. Frischholz and W. Schnell, Compensation of beam loading in the ISR RF cavities, IEEE Trans. Nucl. Sci. 24, 1683 (1977).

[19] F. Pedersen, A novel rf cavity tuning feedback scheme for heavy beam loading, IEEE Trans. Nucl. Sci. 32, 2138 (1985).

[20] R. Garoby et al., RF system for high intensity acceleration in the CERN PS, in Proceedings of the 1989 Particle Accelerator Conference, Chicago, IL (IEEE, New York, 1989).

[21] J. M. Brennan, The upgraded RF system for the AGS and high intensity proton beams, in Proceedings of the Particle Accelerator Conference, Dallas, TX, 1995 (IEEE, New York, 1995), pp. 1489-1493.
[22] M. Paoluzzi et al., Studies on a wideband, solid-state driven rf system for the CERN PS booster, in Proceedings of the 3rd International Particle Accelerator Conference, New Orleans, LA, 2012 (IEEE, Piscataway, NJ, 2012), pp. 3749-3751.

[23] M. Paoluzzi et al., Beam tests using a wide band rf system prototype in the CERN PS booster, in Proceedings of the 6th International Particle Accelerator Conference (IPAC'15), Richmond, VA, 2015, International Particle Accelerator Conference No. 6 (JACoW, Geneva, 2015), pp. 3134-3137.

[24] F. Tamura, M. Yamamoto, C. Ohmori, A. Schnase, M. Yoshii, M. Nomura, M. Toda, T. Shimada, K. Hara, and K. Hasegawa, Multiharmonic rf feedforward system for beam loading compensation in wide-band cavities of a rapid cycling synchrotron, Phys. Rev. ST Accel. Beams 14, 051004 (2011).

[25] F. Tamura, H. Hotchi, A. Schnase, M. Yoshii, M. Yamamoto, C. Ohmori, M. Nomura, M. Toda, T. Shimada, K. Hasegawa, and K. Hara, High intensity single bunch operation with heavy periodic transient beam loading in wide band rf cavities, Phys. Rev. ST Accel. Beams 18, 091004 (2015).

[26] R. Garoby, Low level rf and feedback, in Frontiers of Accelerator Technology, Proceedings of the US-CERNJapan International School (World Scientific, Singapore, 1996), pp. 455-489.

[27] D. Wildman, Transient beam loading compensation in the Fermilab Main Ring, IEEE Trans. Nucl. Sci. 32, 2150 (1985).

[28] LTspice, https://www.analog.com/en/design-center/design -tools-and-calculators/ltspice-simulator.html.

[29] M. Yamamoto, M. Nomura, T. Shimada, F. Tamura, K. Hara, K. Hasegawa, C. Ohmori, M. Toda, M. Yoshii, and A. Schnase, Vacuum tube operation analysis under multiharmonic driving and heavy beam loading effect in J-PARC RCS, Nucl. Instrum. Methods Phys. Res., Sect. A 835, 119 (2016).

[30] A. Schnase et al., MA cavities for J-PARC with controlled Q-value by external inductor, in Proceedings of the 22nd Particle Accelerator Conference, PAC-2007, Albuquerque, NM (IEEE, New York, 2007), pp. 2131-2133.

[31] G. Favia, Study of the beam-cavity interaction in the CERN PS $10 \mathrm{MHz}$ cavities and investigation of hardware solutions to reduce beam loading, Ph.D thesis, Sapienza University, CERN-THESIS-2017-166, 2017.

[32] PIGMG, MicroTCA, https://www.picmg.org/openstandards/ microtca/.

[33] F. Tamura, Y. Sugiyama, M. Yoshii, and M. Ryoshi, Development of next-generation LLRF control system for J-PARC rapid cycling synchrotron, IEEE Trans. Nucl. Sci. 66, 1242 (2019).

[34] F. Tamura, A. Schnase, and M. Yoshii, Dual-harmonic auto voltage control for the rapid cycling synchrotron of the Japan Proton Accelerator Research Complex, Phys. Rev. ST Accel. Beams 11, 072001 (2008).

[35] M. Lonza, Multi-bunch feedback systems, in Proceedings of the CAS-CERN Accelerator School: Course on Digital Signal Processing, Sigtuna, Sweden (CERN-2008-003) 
(2007), pp. 285-330, https://doi.org/10.5170/CERN-2008 $-003.285$

[36] M. P. Donadio, CIC filter introduction, http://dspguru.com/ files/cic.pdf.

[37] F. Tamura, A prototype system of multiharmonic vector voltage control for the J-PARC rapid cycling synchrotron, in Proceedings of the 2017 Low Level RF Workshop, Barcelona, Spain (ALBA, Barcelona, Spain, 2017), pp. O-20.

[38] J. Molendijk, Introducing fixed frequency clock operation on the CERN VXS LLRF platform, in Proceedings of the 2017 Low Level RF Workshop, Barcelona, Spain (ALBA, Barcelona, Spain, 2017), pp. 0-22.

[39] A. Schnase, M. Nomura, F. Tamura, M. Yamamoto, S. Anami, E. Ezura, K. Hara, C. Ohmori, A. Takagi, and M. Yoshii, Cascaded integrator comb filters with smoothly varying coefficients for reduced delay in synchrotron feedback loops, Phys. Rev. Accel. Beams 8, 122001 (2005).
[40] F. Tamura, Y. Sugiyama, M. Yoshii, C. Ohmori, M. Yamamoto, T. Shimada, M. Nomura, K. Hasegawa, K. Hara, and M. Furusawa, Baseband simulation model of the vector rf voltage control system for the J-PARC RCS, J. Phys. Conf. Ser. 1067, 072030 (2018).

[41] F. Tamura et al., Longitudinal painting with large amplitude second harmonic rf voltages in the rapid cycling synchrotron of the Japan Proton Accelerator Research Complex, Phys. Rev. Accel. Beams 12, 041001 (2009).

[42] F. Tamura, M. Yoshii, A. Schnase, C. Ohmori, M. Yamamoto, M. Nomura, M. Toda, T. Shimada, K. Hara, and K. Hasegawa, Achievement of very low jitter extraction of high power proton beams in the J-PARC RCS, Nucl. Instrum. Methods Phys. Res., Sect. A 647, 25 (2011).

[43] M. Angoletta et al., Control and operation of a wideband $\mathrm{rf}$ system in CERN's PS booster, in Proceedings of the International Particle Accelerator Conference (IPAC'17), Copenhagen (JACoW, Geneva, 2017), pp. 4050-4053. 